\title{
The Multi-Artery Fractional Flow Reserve (FFR) Method in The Percutaneous Coronary Intervention (PCI) Practice
}

\author{
Ilan A Yaeger* \\ Department of Cardiology, Israel
}

*Corresponding author: Ilan A Yaeger, Department of Cardiology, Israel, Email: ilan.yaeger41@gmail.com

\author{
Received Date: March 11, 2020
}

Published Date: April 13, 2020

\begin{abstract}
Current single-artery FFR-oriented coronary stenosis severity assessment methods (resting $\mathrm{P}_{d} / \mathrm{P}_{\mathrm{a}^{\prime}}$ wave-free iFR and hyperemic FFR) are used successfully in single vessel disease (SVD) cases. In such cases the stenotic artery is in an independent stand-alone position with a proximal intracoronary aortic driving pressure. The treatment decision criteria of each method (FFR threshold value and FFR 'grey range') apply to FFR of the artery (denoted FFR ${ }^{\text {true }}$ ) which is the remnant fraction left in the stenotic artery of the calculated virtual blood flow of the very same artery prior to the onset of stenosis. As FFR ${ }^{\text {true }}$ can be expressed in terms of the total stenotic resistance $\mathrm{R}_{\mathrm{s}}$ of the artery and the microvascular resistance Rmv associated with the artery, FFR ${ }^{\text {true }}$ can be also justifiably regarded as an ad-hoc intrinsic property of the stenotic artery. It doesn't change unless the artery undergoes revascularization, turning its FFR ${ }^{\text {true }}$ to nearly 1.00. The general scenario however encountered in the PCI practice is one in which a stenotic artery interconnects with other stenotic arteries and it is no longer in an independent stand-alone position since inter-arterial stenosisstenosis interactions take place. Due to this substantial change of circumstances, treatment decision criteria no longer apply to FFR ${ }^{\text {true }}$ of an artery, rather to its actual FFR (denoted FFR ${ }^{\text {real}) \text {. }}$

The multi-artery FFR method is not intended to constitute a substitute for any of the current FFR-oriented methods. As single-artery FFRoriented methods cannot resolve complex scenarios of interacting stenotic coronary arteries, in this article the novel multi-artery FFR method extends these methods to the multi-artery domain with no need to alter their associated experimental techniques nor their treatment decision criteria. Reduction of the mathematics to minimal number of simple formulas in this article enables the PCI practitioner to apply the formulas to measured intracoronary pressures in real time.
\end{abstract}

Keywords: Multi-artery fractional flow reserve; Fractional flow reserve; Percutaneous coronary intervention; Revascularization; Percutaneous transluminal coronary angioplasty

Abbreviations: FFR: Fractional Flow Reserve; PCI: Percutaneous Coronary Intervention; iFR: Instantaneous Wave-Free Ratio; CABG: Coronary Artery Bypass Graft; ISR: In-Stent Restenosis; DES: Drug-Eluting Stent; ST: Stent Thrombosis; MACE: Major Adverse Coronary Event; MVD: MultiVessel Disease; SVD: Single Vessel Disease

\section{Introduction}

\subsection{Single-artery FFR-oriented methods}

Upon encountering a coronary stenosis, the percutaneous coronary intervention (PCI) practitioner is faced with a familiar dilemma: Revascularization or Conservative Medical Treatment? An unjustified revascularization can expose the patient unnecessarily to risks. The risks of a coronary artery bypass graft (CABG) operation are well known. Stenting however has its own risks. Though in-stent restenosis (ISR) has been substantially diminished by using drug-eluting stents (DES), it has not been eradicated.
Within a period of a year or two, in cases of simple lesions and without particular risk factors, the ISR rate is usually less than $5 \%$ [1]. In cases of complex lesions and additional risk factors however, double digit rates are not uncommon [2]. The sudden incidence of stent thrombosis (ST) is around 1\% [3]. By the traditional PCI method, in each of the two extreme cases, the very low and the very high stenosis severity ranges, the decision from which the patient can benefit is obvious from visual inspection and can be readily made. It is in the intermediate stenosis severity range (30\%-70\% 
diameter stenosis) where visual stenosis severity assessment can be difficult due to the absence of a well-defined quantitative criterion. The PCI terrain was consequently ripe for the appearance of some FFR-oriented methods.

At the time of its inception, the hyperemic FFR method has conceptually revolutionized the field of coronary stenosis severity assessment [4]. In contrast to the traditional and essentially qualitative angiographic approach, the FFR method focused on a quantitative measure of the functional performance of the stenotic artery in a pharmacologically induced state of hyperemia. In this method the hyperemia state is mandatory, and the resulting microvascular resistance is minimal and stable while maximal dilatation of the coronary artery and of its associated microvasculature takes place. The hyperemic FFR index of a stenotic coronary artery is the currently available remnant fraction of the (calculated) original maximal blood flow through the artery in its virtual stenosis-free state, namely

$$
\mathrm{FFR}=\mathrm{Q}_{\mathrm{s}} / \mathrm{Q}_{\mathrm{o}}
$$

$\mathrm{Q}_{\mathrm{s}}$ - blood flow through artery in stenotic state

$\mathrm{Q}_{0}$ - blood flow through artery in a virtual stenosis-free state

As the hyperemic pressure and blood flow are in a linearity relationship, the FFR index is obtained experimentally from measurements of intracoronary pressures. If a single stenotic artery is exposed to the aortic pressure, and the venous pressure is assumed to be nearly zero, its hyperemic FFR index (denoted $\mathrm{FFR}^{\text {true}}$ ) is equal numerically to the ratio of the mean distal pressure $\mathrm{P}_{\mathrm{d}}$ and the mean proximal aortic pressure $\mathrm{P}_{\mathrm{a}}[4]$, namely

$$
\mathrm{FFR}^{\text {true }}=\mathrm{P}_{\mathrm{d}} / \mathrm{P}_{\mathrm{a}}
$$

FFR $^{\text {true }}$ of a single stenotic artery can be also expressed in terms of the total stenotic resistance Rs of the artery and its associated microvascular resistance $\mathrm{R}_{\mathrm{mv}}$ :

$$
\mathrm{FFR}^{\text {true }}=1 /\left(1+\mathrm{R}_{\mathrm{s}} / \mathrm{R}_{\mathrm{mv}}\right)
$$

In an experimental statistical study FFR-guided decision for treatment of stenotic coronary arteries proved to be superior to angiography-guided approach when the number of major adverse coronary events (MACE) over a period of several years was taken into consideration [4]. It was also found experimentally that for a single stenotic artery a condition of $\mathrm{FFR}^{\text {true }}<0.75$ implies a mandatory revascularization of the stenotic artery whereas $0.85<\mathrm{FFR}^{\text {true }}$ indicates that treating the single artery just by medication is enough. If the $\mathrm{FFR}^{\text {true }}$ index is in the 'grey range' of treatment decision uncertainty, $0.75 \leq \mathrm{FFR}^{\text {true }} \leq 0.85$, additional clinical factors related to the patient under consideration should be considered in order to reach the correct treatment decision [5]. Patients feel uncomfortable during the state of hyperemia associated with the hyperemic FFR method. In order to get around this negative aspect and other downsides of this method, the instant wave-free ratio (iFR) method in which no state of hyperemia is required was introduced in 2012 [6]. The familiar resting $\mathrm{P}_{d} /$
$\mathrm{P}_{\mathrm{a}}$ method in which the ratio distal to aortic pressure at rest is averaged over the full cardiac cycle (for several consecutive cycles) also shows results comparable to these of the hyperemic FFR and free wave iFR methods [7].

Despite the establishment of the superiority of the hyperemic FFR method over angiographic stenosis severity assessment in the FAME study [8], there is a grave downside associated with the 3 FFR-oriented methods - they do not take into account inter-arterial interactions as can be seen in the FAME study itself. The cases treated in that study were formally multi vessel disease (MVD) cases involving 2 or 3 major coronary arteries LAD, LCx and RCA. However, an inspection of the conditions of the FAME study reveals that cases of diseased LMCA were excluded from the study, leaving each of LAD and LCx arteries directly exposed to the aortic pressure. This fact rendered all 3 arteries independent of each other since the proximal pressure in each one was the full aortic pressure, and each was consequently treated in that study as an independent single vessel disease (SVD) case. The inevitable conclusion is that the basic hyperemic FFR method cannot yield a rigorous resolution of complex MVD cases. In recent years attempts have been made to study the effect of downstream stenoses on LMCA employing the hyperemic single-artery FFR method but since this method does not take into account inter-arterial interactions, those attempts provided only general quantitative results rather than rigorous resolution of stenotic coronary configurations involving LMCA on an individual case basis $[9,10]$.

\subsection{Multi-artery FFR method}

The multi-artery FFR method made its first appearance online at the end of 2015 [11]. The method is based essentially on the familiar Kirchhoff's laws of analogous direct-current circuits. It considers inter-arterial interactions and has demonstrated its capability to resolve a variety of 2-artery and 3-artery stenotic coronary configurations [12]. The method does not constitute a substitute for any of the current 3 single-artery diagnostic methods, it just provides the correct pressure-flow relationships in coronary configurations encountered in the PCI practice. As for the treatment-decision criteria of the multi-artery FFR method used in this article, a cut-off value of FFR $<0.75$ with a 'grey range' of treatment-decision uncertainty of $0.75 \leq$ FFR $\leq 0.85$, which are usually associated with the hyperemic single-artery FFR method, have been chosen. This choice of treatment-decision criteria has been made just for the establishment of a common basis for comparison purposes, but it does not imply in any way that the hyperemic FFR method is superior to the iFR or to the resting $\mathrm{P}_{\mathrm{d}} /$ $\mathrm{P}_{\mathrm{a}}$ methods.

At this point it should be stressed that when a stenotic coronary artery is just by itself, in a stand-alone position, without possible influence by interconnected stenotic arteries, then the treatment decision criteria (FFR cut-off value and FFR 'grey range') apply to its FFR ${ }^{\text {true. }}$. However when there are other stenotic arteries in connection with the stenotic artery under consideration, the flow 
through the stenotic artery is no longer determined just by its total stenotic resistance $\mathrm{R}_{\mathrm{s}}$ and the resistance $\mathrm{R}_{\mathrm{mv}}$ of the microvasculature associated with the artery (like in formula (3) here) but also by the influence of the other stenotic arteries. These arteries impede and reduce the blood flow through the stenotic artery compared to its blood flow in the virtual state of these arteries being stenosisfree. The multi-artery FFR is the appropriate method for resolving such scenarios and the treatment decision criteria no longer apply directly to $\mathrm{FFR}^{\text {true }}$, rather to $\mathrm{FFR}^{\text {real }}$ which is defined as the current remnant fraction left of the original blood flow $Q_{0}$ through the stenotic artery in the virtual state of all arteries being stenosis-free (including the artery under consideration):

$$
\mathrm{FFR}^{\text {real }}=\mathrm{Q}_{\mathrm{s}}^{\text {real }} / \mathrm{Q}_{\mathrm{o}}
$$

$\mathrm{Q}_{\mathrm{s}}{ }^{\text {real }}$ - actual blood flow through the stenotic artery

$Q_{0}$ - blood flow through the artery when all arteries are virtually in stenosis-free state

$\mathrm{FFR}^{\text {real }}$ reflects the condition of the stenotic artery and obviously $\mathrm{FFR}^{\text {real }} \leq \mathrm{FFR}^{\text {true }}$.

This inequality is extremely important to the PCI practitioner. It indicates that $\mathrm{FFR}^{\text {real }}$ of an artery can be improved indirectly by revascularizing other arteries (that are interacting with the artery under consideration) but only up to a certain limit, because FFR $^{\text {true }}$ is the upper bound of FFR real. If it is needed and if feasible, this restriction can be practically lifted by revascularizing the stenotic artery itself, thus turning its FFR ${ }^{\text {true }}$ to nearly $\mathrm{FFR}^{\text {true }}=1.00$ (but even then, it does not necessarily imply $\mathrm{FFR}^{\text {real }}=1.00$ ).

Being a relatively novel method, the multi-artery FFR method deals essentially with the salient features of stenotic coronary configurations. On the grounds of simplicity, it is assumed in the present article that collaterals are not involved with the arteries of these stenotic coronary configurations. At an advanced stage of coronary disease however, collaterals may assume a substantially significant role. The scenarios under consideration in this article are mostly of a stenotic unprotected LMCA with stenoses also in one or two downstream main arteries, occasionally combined with a stenotic small side branch as well as 'mother'-'daughter' configurations and a variety of other equivalent configurations as will be indicated in the various sections of the article.

\section{Methodology - The Basic Essentials}

The full extent of the mathematics associated with the multiartery FFR method will not be presented in this article, it is described in the various articles about the capabilities of the method that have been published in recent years [11-15]. For the convenience of the reader, a concise methodology is given in the supplementary Appendix A of this article. In this paragraph stenotic coronary configurations associated with various scenarios in the PCI practice will be presented and the basic formulas for obtaining their current status and condition (FFR ${ }^{\text {true }}$ and $\mathrm{FFR}^{\text {real }}$ of each artery) will be given in terms of the measured intracoronary pressures at locations marked in the appropriate figures in the article. The condition of each artery is reflected by its FFR $^{\text {real }}$ and it may be improved by revascularizing some other artery that impedes its blood flow. This potential improvement is limited by the inequality $\mathrm{FFR}^{\text {real }} \leq \mathrm{FFR}^{\text {true }}$ which implies that if FFR ${ }^{\text {true }}$ of an artery is low, not much can be gained by revascularizing other arteries and therefore the stenotic artery itself should be revascularized, if technically feasible and if hemodynamically justifiable. The formulas given in this paragraph constitute the first stage of the resolution of the stenotic coronary configuration associated with each of the scenarios presented in this paragraph. Further stages of the resolution process are presented in the supplementary Appendix A of the article. However, to experienced PCI practitioners sometimes the FFR ${ }^{\text {true }}$ and $\mathrm{FFR}^{\text {real }}$ of each artery obtained in the first stage suffice for devising the appropriate strategy for the resolution of the configuration under consideration.

\subsection{3-artery coronary configurations of sizable arteries}

The most common 3-artery configuration of sizable coronary arteries encountered in the PCI practice is the unprotected [LMCA][LCx]-[LAD] configuration (Figure 1). In the general case, each one of the arteries of the configuration is potentially stenotic and the current status and condition of each artery can be obtained from the values of the measured intracoronary pressures at the locations marked in (Figure 1). The current condition of the arteries is given by the following formulas [13]:

$$
\begin{aligned}
& \operatorname{FFR}^{\text {real }}(1)=\left(P_{3 d}+P_{2 d}\right) /\left(2 \cdot P_{a}\right) \\
& \operatorname{FFR}^{\text {real }}(2)=P_{2 d} / P_{a} \\
& \operatorname{FFR}^{\text {real }}(3)=P_{3 d} / P_{a}
\end{aligned}
$$

By the $\mathrm{FFR}^{\text {real }}$ of an artery one can immediately tell if its condition is acceptable or not. Sometimes the condition of the configuration is unacceptable without a noticeable stenotic culprit artery, it is the combined effect of more than just one stenotic artery. It can be occasionally seen that each artery, if it were just by itself (in a standalone position), its condition would be acceptable but due to the interference of other stenotic arteries its blood flow is impeded to an unacceptable level.

The $\mathrm{FFR}^{\text {true }}$ values of the arteries are given by the following formulas [13]:

$$
\begin{aligned}
& \operatorname{FFR}^{\text {true }}(1)=1 /\left\{\left[2 \cdot\left(\mathrm{P}_{\mathrm{a}}-\mathrm{P}_{\mathrm{p}}\right)\right] /\left(\mathrm{P}_{3 \mathrm{~d}}+\mathrm{P}_{2 \mathrm{~d}}\right)+1\right\} \\
& \operatorname{FFR}^{\text {true }}(2)=\mathrm{P}_{2 \mathrm{~d}} / \mathrm{P}_{\mathrm{p}} \\
& \operatorname{FFR}^{\text {true }}(3)=\mathrm{P}_{3 \mathrm{~d}} / \mathrm{P}_{\mathrm{p}}
\end{aligned}
$$

The FFR ${ }^{\text {true }}$ values of the arteries can be very useful to the PCI practitioner for devising a proper resolution strategy, especially by assessing the potential of optional revascularizations considering the inequality FFR ${ }^{\text {real }} \leq \mathrm{FFR}^{\text {true }}$ (see Example 1 in Section 3.1).

It should be noted that sometimes 2-artery scenarios can be resolved by an equivalent 3-artery configuration of (Figure 1). 
Occasionally the first diagonal artery $D_{1}$ is a sizable artery and sometimes the first obtuse marginal artery $\mathrm{OM}_{1}$ happens to be a sizable artery. Under such circumstances, if LMCA is stenosis-free (thus maintaining the aortic pressure as the driving pressure of the configuration), the formulas of this section can be applied for example also to the following 2 configurations:
1) [prox LAD]-[remainder LAD]-[sizable $\left.\mathrm{D}_{1}\right]$

Artery 1=prox LAD; Artery 2=remainder LAD; Artery3=sizable $\mathrm{D}_{1}$

2) [prox LCx]-[remainder LCx]-[sizable $\left.\mathrm{OM}_{1}\right]$

Artery 1=prox LCx; Artery 2=remainder LCx; Artery3=sizable $\mathrm{OM}_{1}$

\section{3-artery configuration}

\section{Pressure-Resistance-Flow scheme}

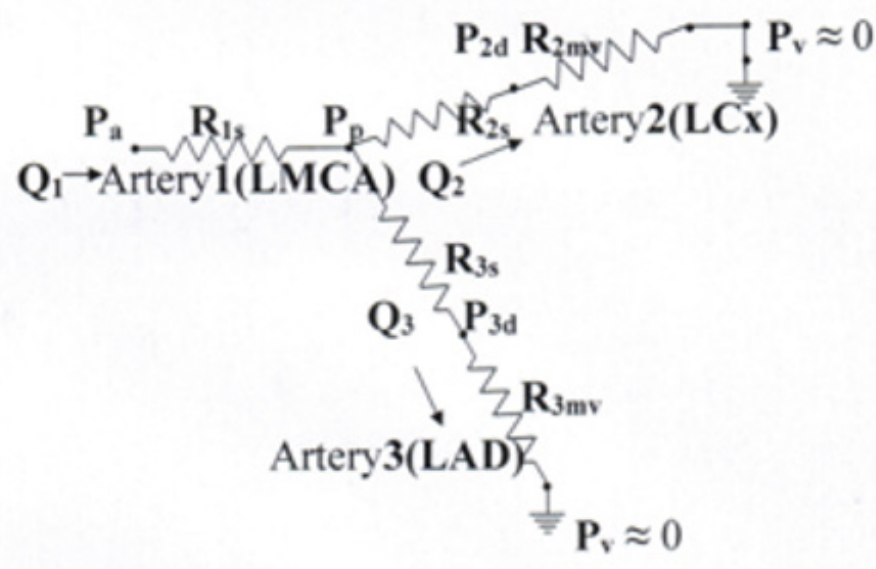

Figure 1: Artery 1 (LMCA) is functionally the conductance artery whereas Artery 2 (LCx) and Artery 3 (LAD) are the end arteries. All arteries of the configuration are potentially stenotic. First subscript of resistance $R_{i t}$ indicates the artery number $(i=1,2,3)$ and the second subscript indicates the type of resistance ( $\mathrm{t}=\mathrm{s}$-stenotic, mv-microvascular). $\mathrm{P}$ and $\mathrm{P}$ are the mean aortic pressure and the nearly zero pressure of the venous bed respectively. $P_{p}$ is the proximal pressure of Artery 2 (LCX) and Artery $3(L A D)$ whereas $P_{2 d}$ and $P_{3 d}$ are the distal pressures of Arteries 2 (LCX) and 3 (LAD) respectively. Qi is the blood flow (volume units per unit of time) in Artery $i(i=1,2,3)$.

\subsection{A 'mother'-'daughter' scenario - resolution by an equivalent 3 -artery configuration}

In this scenario there is a sizable ('mother') artery and its small side branch ('daughter') artery, each one potentially stenotic (Figure 2). Due to size difference, clearly the inter arterial interactions in this configuration are completely different from the interactions in the 3-artery configuration of sizable arteries. On the same grounds, the small side branch is obviously affected by the sizable 'mother' artery but has no influence on the latter. This will manifest itself in the relevant formulas. It should be noted that it is assumed that the arteries preceding the 'mother' artery are stenosis-free so that the aortic pressure is maintained all the way to the proximal segment of the 'mother' artery. In (Figure 2) Artery 1 and Artery 2 constitute the sizable 'mother' artery: Artery 1 is the proximal segment of the 'mother' artery (all the way to the split-off point of the small side branch) whereas Artery 2 is the remainder of the 'mother' artery.
Artery 3 is the small side branch in (Figure 2).

The current condition of the arteries can be obtained from the measured intracoronary pressures at the locations marked in Figure 2 [14]:

$$
\begin{aligned}
& \operatorname{FFR}^{\text {real }}(1)=P_{2 d} / P_{a} \\
& \operatorname{FFR}^{\text {real }}(2)=P_{2 d} / P_{a} \\
& \operatorname{FFR}^{\text {real }}(3)=P_{3 d} / P_{a}
\end{aligned}
$$

The FFR ${ }^{\text {true }}$ values can be also obtained from the measured intracoronary pressures [14]:

$$
\begin{aligned}
& \operatorname{FFR}^{\text {true }}(1)=1 /\left\{\left[\left(\mathrm{P}_{\mathrm{a}}-\mathrm{P}_{\mathrm{p}}\right)\right] / \mathrm{P}_{2 \mathrm{~d}}+1\right\} \\
& \operatorname{FFR}^{\text {true }}(2)=\mathrm{P}_{2 \mathrm{~d}} / \mathrm{P}_{\mathrm{p}} \\
& \operatorname{FFR}^{\text {true }}(3)=\mathrm{P}_{3 \mathrm{~d}} / \mathrm{P}_{\mathrm{p}}
\end{aligned}
$$




\section{Equivalent 3-artery configuration}

\section{Pressure-Resistance-Flow scheme}

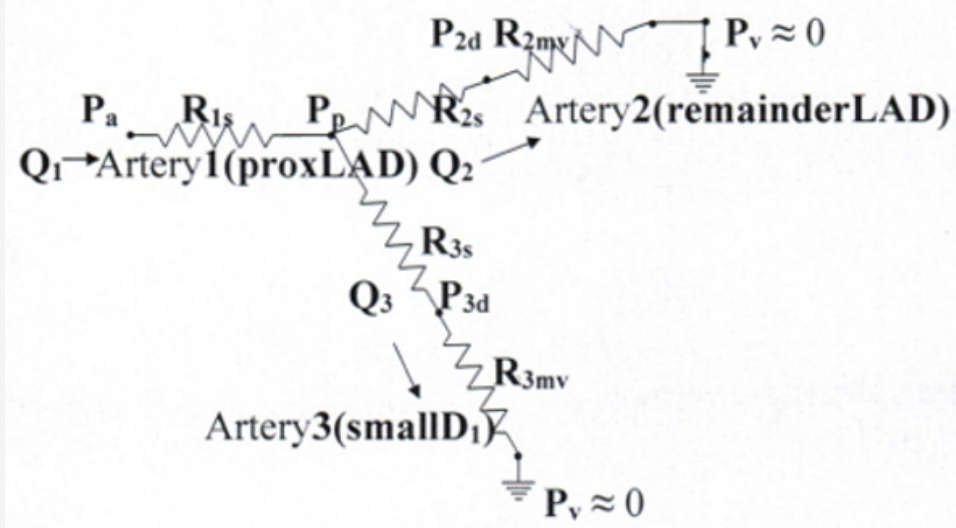

Figure 2: The 3-artery configuration represents a 'mother'-'daughter' configuration composed of a potentially stenotic LAD artery and its potentially stenotic first diagonal $D_{1}$ side branch. The LAD artery is preceded by a non-stenotic LMCA and the aortic pressure is therefore maintained all the way to the proximal part of the LAD artery. The LAD artery is represented in the arterial scheme by 2 arteries, Artery 1 represents the proximal part of LAD artery whereas Artery 2 represents the remainder of the LAD artery. The side branch $D_{1}$ is represented by Artery 3 . First subscript of resistance $R_{i t}$ indicates the artery number $(i=1,2,3)$ while the second subscript indicates the type of resistance ( $t=s$-stenotic, mv-microvascular). $P_{a}$ and $P_{v}$ are the mean aortic pressure and the nearly zero pressure of the venous bed respectively. $P_{p}$ is the proximal pressure in Artery 2 (remainderLAD) and Artery $3\left(D_{1}\right)$ whereas $P_{2 d}$ and $P_{3 d}$ are the distal pressures in Arteries 2 (remainderLAD) and $3\left(D_{1}\right)$ respectively. $Q_{i}$ is the blood flow (volume units per unit of time) in Artery $i(i=1,2,3)$. In the analysis of the MVD scenario in the text, Artery 1 (proxLAD) is functionally the conductance artery, Artery 2 (remainderLAD) and Artery $3\left(\mathrm{D}_{1}\right)$ are end arteries.

As indicated already, $\mathrm{FFR}^{\text {true }}$ of an artery does not change unless the artery is revascularized, in which case FFR ${ }^{\text {true }}$ becomes nearly 1.00. The PCI practitioner can use the $\mathrm{FFR}^{\text {true }}$ values for assessment of possible outcomes of optional revascularizations (Example2 in Section 3.2).

\subsection{A complex 4-artery configuration}

The configuration of Figure 3 consists of the familiar 3 sizable arteries, unprotected LMCA, LCX and LAD with an additional small first diagonal side branch $D_{1}$. Due to the substantial differences in size, the sizable arteries can affect $\mathrm{D}_{1}$ but $\mathrm{D}_{1}$ cannot affect LAD nor any of the other sizable arteries. After performing intracoronary pressure measurements, the first step that the PCI practitioner must take in order to assess the current condition of each artery is calculating its FFR ${ }^{\text {real }}$ from the measured intracoronary pressures by the following formulas $[13,14]$ (see Figure 3):

$F^{2} R^{\text {real }}(1)=\left(\mathrm{P}_{3 \mathrm{~d}}+\mathrm{P}_{2 \mathrm{~d}}\right) /\left(\mathrm{P}_{\mathrm{a}} \cdot 2\right) ; \mathrm{FFR}^{\text {real }}(2)=\mathrm{P}_{2 \mathrm{~d}} / \mathrm{P}_{\mathrm{a}^{\prime}} ; \mathrm{FFR}^{\text {real }}(3)=\mathrm{P}_{3 \mathrm{~d}} / \mathrm{P}_{\mathrm{a}^{\prime}} \mathrm{FFR}^{\text {real }}(4)=\mathrm{P}_{4 \mathrm{~d}} / \mathrm{P}_{\mathrm{a}}$;

In order to make initial assessments regarding the outcomes of potential revascularizations, the PCI practitioner should calculate the current FFR ${ }^{\text {true }}$ values for each artery in the configuration by the following formulas [13] (Figure 3):

$$
\begin{aligned}
& \operatorname{FFR}^{\text {true }}(1)=1 /\left\{\left[\left(\mathrm{P}_{\mathrm{a}}-\mathrm{P}_{\mathrm{p}}\right) \cdot 2\right] /\left(\mathrm{P}_{3 \mathrm{~d}}+\mathrm{P}_{2 \mathrm{~d}}\right)+1\right\} ; \mathrm{FFR}^{\text {true }}(2)=\mathrm{P}_{2 \mathrm{~d}} / \mathrm{P}_{\mathrm{p}} \\
& \operatorname{FFR}^{\text {true }}(3)=\mathrm{P}_{3 \mathrm{~d}} / \mathrm{P}_{\mathrm{p}} ; \mathrm{FFR}^{\text {true }}(4)=\mathrm{P}_{4 \mathrm{~d}} / \mathrm{P}_{3 \mathrm{~d}} ;
\end{aligned}
$$

As indicated already, the FFR ${ }^{\text {true }}$ of an artery remains constant unless the artery is revascularized, turning its FFR ${ }^{\text {true }}$ to nearly 1.00. Note that $\mathrm{FFR}^{\text {real }} \leq \mathrm{FFR}^{\text {true }}$. This inequality states the simple fact that the blood flow through a stenotic coronary artery is highest when the artery is just by itself in the absence of interconnected stenotic arteries the interference of which can possibly impede and reduce the blood flow through the artery. FFR ${ }^{\text {true }}$ therefore indicates the maximal FFR ${ }^{\text {real }}$ that the artery can have under best conditions of no interference at all and being exposed to aortic pressure. A low value of FFR ${ }^{\text {true }}$ implies that the artery itself may be the culprit for its possibly unacceptable condition, therefore other directions of seeking an optimal resolution may deserve a lower priority. In the scenario described here, the 3 sizable arteries dominate the scene, therefore the partial 3-artery configuration [LMCA][LCx]-[LAD] (Figure 1) should be resolved first (Section 3.1) and only then the attention should turn to the small side branch $\mathrm{D}_{1}$. Once the PCI practitioner calculates the outcomes of the desired revascularizations within the 3-artery configuration of the sizable arteries (FFR ${ }^{\text {true }}$ and FFR $^{\text {real }}$ of each artery), their anticipated effect on the small side branch $\mathrm{D}_{1}$ should be figured out. As can be seen by the formulas in this section, $\mathrm{FFR}^{\text {true }}$ and $\mathrm{FFR}^{\text {real }}$ of $\mathrm{D}_{1}$ are dependent on the intracoronary pressures at specific locations marked in (Figure 3). One needs to calculate the intracoronary pressures anticipated in the wake of the revascularizations planned by the PCI practitioner for the resolution of the 3-artery configuration of the sizable arteries. The anticipated intracoronary pressures in terms of FFR ${ }^{\text {true }}$ of the arteries are the following (Appendix A): 
$P_{p}=\left[2 \cdot P_{a} \cdot F F R^{\text {true }}(1)\right] /\left\{\left[1-F F R^{\text {true }}(1)\right] \cdot\left[F F R^{\text {true }}(2)+F^{\text {true }}(3)\right]\right.$ $\left.+2 \cdot \mathrm{FFR}^{\text {true }}(1)\right\}$

$\mathrm{P}_{2 \mathrm{~d}}=\left[2 \cdot \mathrm{P}_{\mathrm{a}} \cdot \mathrm{FFR}^{\text {true }}(1) \cdot \mathrm{FFR}^{\text {true }}(2)\right] /\left\{\left[1-\mathrm{FFR}^{\text {true }}(1)\right] \cdot\left[\mathrm{FFR}^{\text {true }}(2)\right.\right.$ $\left.\left.+\mathrm{FFR}^{\text {true }}(3)\right]+2 \cdot \mathrm{FFR}^{\text {true }}(1)\right\}$

$P_{3 d}=\left[2 \cdot P_{a} \cdot F^{2} R^{\text {true }}(1) \cdot F^{\text {true }}(3)\right] /\left\{\left[1-F^{2} R^{\text {true }}(1)\right] \cdot\left[F F R^{\text {true }}(2)\right.\right.$ $\left.\left.+\mathrm{FFR}^{\text {true }}(3)\right]+2 \cdot \mathrm{FFR}^{\text {true }}(1)\right\}$

$\mathrm{P}_{4 \mathrm{~d}}=\left[2 \cdot \mathrm{P}_{\mathrm{a}} \cdot \mathrm{FFR}^{\text {true }}(1) \cdot \mathrm{FFR}^{\text {true }}(3) \cdot \mathrm{FFR}^{\text {true }}(4)\right] /\left\{\left[1-\mathrm{FFR}^{\text {true }}(1)\right] \cdot\right.$ $\left.\left[\mathrm{FFR}^{\text {true }}(2)+\mathrm{FFR}^{\text {true }}(3)\right]+2 \cdot \mathrm{FFR}^{\text {true }}(1)\right\}$
It should be noted that from the formulas it is obvious that FFR $^{\text {true }}$ of $\mathrm{D}_{1}$ (Artery 4 in (Figure 3 )) does not affect the intracoronary pressures $\mathrm{P}_{p}, \mathrm{P}_{2 d}$ and $\mathrm{P}_{3 d}$ within the 3 -artery configuration of sizable arteries but $\mathrm{P}_{4 \mathrm{~d}}$ within the side branch $\mathrm{D}_{1}$ is affected by all the arteries. The predicted values of $\mathrm{FFR}^{\text {true }}$ and $\mathrm{FFR}^{\text {real }}$ of $\mathrm{D}_{1}$ resulting from the implementation of some of the optional revascularizations can be obtained from the expressions for $\mathrm{P}_{3 \mathrm{~d}}$ and $\mathrm{P}_{4 \mathrm{~d}}$ because one has:

$\operatorname{FFR}^{\text {true }}(4)=\mathrm{P}_{4 \mathrm{~d}} / \mathrm{P}_{3 \mathrm{~d}} ; \operatorname{FFR}^{\text {real }}(4)=\mathrm{P}_{4 \mathrm{~d}} / \mathrm{P}_{\mathrm{a}} ;$

\section{4-Artery Coronary Configuration}

\section{Pressure-Resistance-Flow Scheme}

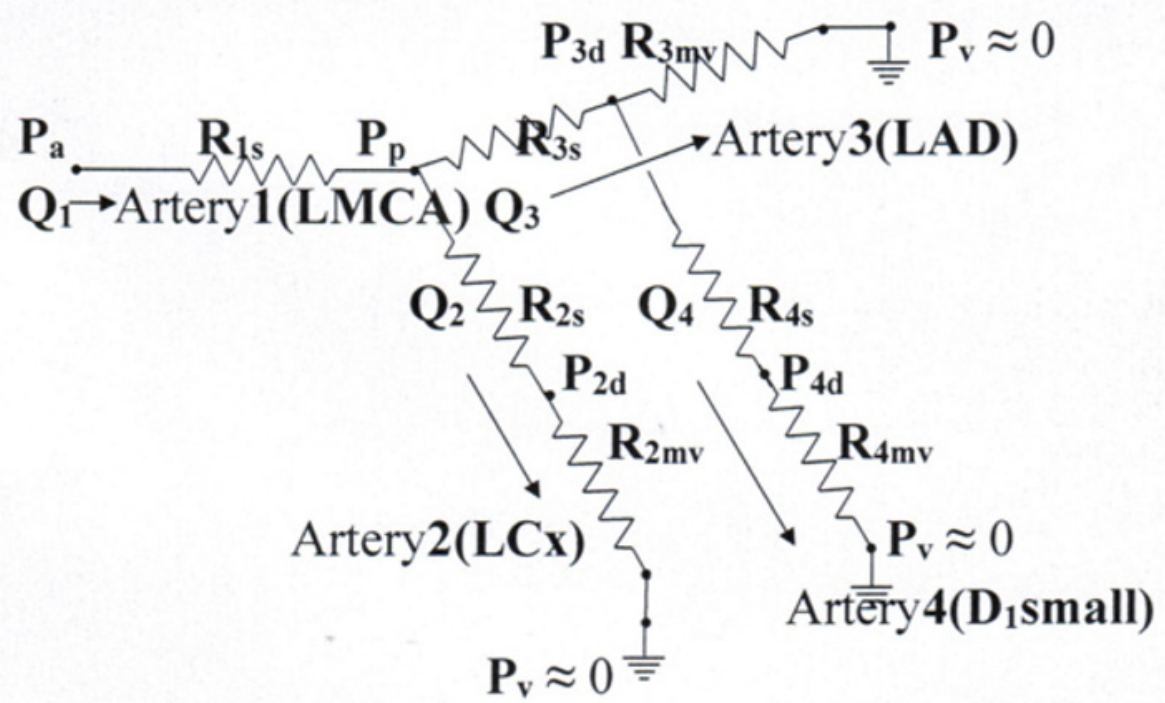

Figure 3: Artery 1 (LMCA) is functionally the conductance artery whereas Artery 2 (LCx) and Artery 3 (LAD) are end arteries that lead to the myocardium. Artery $4\left(D_{1}\right)$ is a small side-branch of Artery $3(L A D)$ that also leads to the myocardium. All the arteries of the configuration are potentially stenotic. First subscript of resistance $R_{i t}$ of artery $i$ indicates the artery number $(i=1,2,3,4)$ while the second subscript indicates the type of resistance ( $\mathrm{t}=\mathrm{s}$-stenotic, mv-microvascular). $\mathrm{P}_{\mathrm{f}}$ and $\mathrm{P}_{\mathrm{v}}$ are the mean aortic pressure and the nearly zero pressure of the venous bed respectively. $P_{p}$ is the proximal pressure of Artery 2 (LCX) and Artery 3 (LAD) while $P_{2 d}, P_{3 d}$ and $P_{4 d}$ are the distal pressures of Artery 2 (LCx), Artery 3 (LAD) and Artery 4 (D1) respectively. Qi is the blood flow (volume units per unit of time) in Artery i $(i=1,2,3,4)$.

In Section 3.3 of this article Example 3 of a resolution of a 4-artery stenotic coronary configuration (Figure 3 ) by the multi-artery FFR method employing the formulas of the present section and simulated intracoronary pressures is presented in detail.

\section{Results -Numerical Simulation Examples}

\subsection{Example \#1 - Stenotic 3-artery configuration of siz- able arteries (Figure 1)}

The coronary configuration of (Figure 1) is (1) LMCA - (2) LCx - (3) LAD

In this configuration of sizable arteries each artery is potentially stenotic. The simulated intracoronary pressures in this case are the following (in units of $\mathrm{mmHg}$ ):

$$
P_{a}=100 ; P_{p}=85 ; P_{2 d}=70 ; P_{3 d}=75
$$

Calculated present status of the arteries:

$\operatorname{FFR}^{\text {true }}(1)=0.83 ; \operatorname{FFR}^{\text {true }}(2)=0.82 ; \operatorname{FFR}^{\text {true }}(3)=0.88$; (by formulas $(8)-(10)$ )

$\operatorname{FFR}^{\text {real }}(1)=0.73 ; F^{\text {real }}(2)=0.70 ; F^{\text {real }}(3)=0.75$; (by formulas $\left.(5)-(7)\right)$

The FFR $^{\text {true }}$ values of the arteries here indicate that if each artery had been the only stenotic artery around, its condition would have been acceptable. The inter-arterial stenosis-stenosis interaction however severely impedes the blood flow through the configuration and renders its condition unacceptable. Obviously, revascularization is in order, but strategy is important too. Revascularization of LMCA, if considered by the PCI practitioner, should be usually carried out by a CABG operation (unless it is contraindicated due to circumstances). This will consequently not be the PCI practitioner's first choice. Turning to the other two arteries, LCX and LAD, in search for a 'candidate' for revascularization, the 
practitioner should take a good look at the FFR ${ }^{\text {true }}$ of each artery. It is true that the condition of an artery in a configuration is reflected by its $F_{F R}{ }^{\text {real }}$, not by its $F_{F R}^{\text {true }}$, but it is the inequality $F^{\text {real }} \leq$ FFR $^{\text {true }}$ that one needs to consider. If the number of stenotic arteries connected to an artery decreases (by revascularization), its $\mathrm{FFR}^{\text {real }}$ increases but the inequality indicates that this increase is limited since FFR ${ }^{\text {true }}$ of an artery constitutes the upper bound of $\mathrm{FFR}^{\text {real }}$. A glance at FFR ${ }^{\text {true }}$ of $\mathrm{LCx}$ and LAD shows that FFR ${ }^{\text {real }}$ of $\mathrm{LCx}$ is more restricted than FFR ${ }^{\text {real }}$ of LAD (because $F_{F R}^{\text {true }}(2)<F^{\text {true }}(3)$ ). It seems therefore more beneficial to revascularize $\mathrm{LCx}$, increasing its smaller FFR ${ }^{\text {true }}$ to nearly FFR $^{\text {true }}(2)=1.00$.

The post-revascularization status of the arteries would be

$$
\operatorname{FFR}^{\text {true }}(1)=0.83 ; \operatorname{FFR}^{\text {true }}(2)=1.00 ; \operatorname{FFR}^{\text {true }}(3)=0.88
$$

$\mathrm{FFR}^{\text {real }}(1)=0.79 ; \mathrm{FFR}^{\text {real }}(2)=0.84 ; \mathrm{FFR}^{\text {real }}(3)=0.74$; (by formulas (A29) - (A31) of Appendix A)

It is evident that the condition of LAD artery is unacceptable. The condition of LAD has deteriorated since the blood flow in LCX competes with the blood flow in LAD. With the reduction of the stenosis in LCx to zero, increase of the blood flow in LCx results in some decrease of the flow in LAD.

It seems that LAD should be revascularized too. Revascularization of both LCX and LAD arteries yields the following results:

$$
\operatorname{FFR}^{\text {true }}(1)=0.83 ; \operatorname{FFR}^{\text {true }}(2)=1.00 ; \operatorname{FFR}^{\text {true }}(3)=1.00
$$

$\mathrm{FFR}^{\text {real }}(1)=0.83 ; \mathrm{FFR}^{\text {real }}(2)=0.83 ; \mathrm{FFR}^{\text {real }}(3)=0.83$; (by formulas (A29) - (A31) of Appendix A)

Evidently, the condition of the arteries of the configuration is acceptable and no revascularization of LMCA is required.

\subsection{Example \#2 - A 'mother'-'daughter' scenario re- solved by an equivalent 3-artery configuration (Figure 2) of Section 2.2}

The coronary configuration of (Figure 2) is (1) proxLAD - (2) remainderLAD - (3) smallD

In this scenario the 'mother' artery is the LAD artery preceded by a non-stenotic LMCA. The aortic pressure $\mathrm{P}_{\mathrm{a}}$ is therefore maintained all the way to the proximal segment of LAD. The LAD artery in the scheme of (Figure 2) is a combination of Artery 1 which is the proximal part of the LAD artery and Artery 2 which is the remainder of the LAD artery. It should be noted that each part of the LAD artery may be stenotic. Artery 3 represents the optionally stenotic small 'daughter' artery $\mathrm{D}_{1}$. The simulated intracoronary pressures in this example (in units of $\mathrm{mmHg}$ ) are the following:

$\mathrm{P}_{\mathrm{a}}=105 ; \mathrm{P}_{\mathrm{p}}=85 ; \mathrm{P}_{2 \mathrm{~d}}=70 ; \mathrm{P}_{3 \mathrm{~d}}=75$

Calculated present status of the arteries:

$\operatorname{FFR}^{\text {true }}(1)=0.78 ; \operatorname{FFR}^{\text {true }}(2)=0.82 ; F^{\text {true }}(3)=0.88$; (by formulas $(14)-(16)$ ) $F R^{\text {real }}(1)=0.67 ; F F R^{\text {real }}(2)=0.67 ; F F R^{\text {real }}(3)=0.71 ;($ by formulas $(11)-(13))$
It is obvious that the condition of both the 'mother' artery and the 'daughter' artery is unacceptable. In devising the proper strategy to resolve this scenario, the PCI practitioner has to take into account that due to the substantial difference in size, an event that takes place in the 'mother' artery affects also the condition of the 'daughter' artery but the opposite is not true. Namely, the 'mother' artery is not affected by whatever takes place in the 'daughter' artery. Also, it is obvious that stenting the proximal section of LAD artery (Artery 1 in Figure 2) exposes the remainder of LAD artery, as well as the $\mathrm{D}_{1}$ artery, to the aortic pressure. In such a case, just by the definitions of FFR ${ }^{\text {true }}$ and FFR ${ }^{\text {real }}$ and without any further calculations one has:

$$
\begin{aligned}
& \operatorname{FFR}^{\text {true }}(1)=1.00 ; \operatorname{FFR}^{\text {true }}(2)=0.82 ; \operatorname{FFR}^{\text {true }}(3)=0.88 \\
& \operatorname{FFR}^{\text {real }}(1)=\operatorname{FFR}^{\text {true }}(2)=0.82 \\
& \operatorname{FFR}^{\text {real }}(2)=\mathrm{FFR}^{\text {true }}(2)=0.82 \\
& \operatorname{FFR}^{\text {real }}(3)=\operatorname{FFR}^{\text {true }}(3)=0.88
\end{aligned}
$$

The condition of both the 'mother' and 'daughter' arteries is acceptable but should the PCI practitioner desire to further improve the condition of the LAD artery, the remainder of the LAD artery can be stented too, yielding (just by the definitions of FFR ${ }^{\text {true }}$ and $\mathrm{FFR}^{\text {real }}$ ):

$$
\begin{aligned}
& \operatorname{FFR}^{\text {true }}(1)=1.00 ; \mathrm{FFR}^{\text {true }}(2)=1.00 ; \mathrm{FFR}^{\text {true }}(3)=0.88 \\
& \operatorname{FFR}^{\text {real }}(1)=\mathrm{FFR}^{\text {true }}(1)=\mathrm{FFR}^{\text {true }}(2)=1.00 \\
& \operatorname{FFR}^{\text {real }}(2)=\mathrm{FFR}^{\text {true }}(1)=\mathrm{FFR}^{\text {true }}(2)=1.00 \\
& \operatorname{FFR}^{\text {real }}(3)=\mathrm{FFR}^{\text {true }}(3)=0.88
\end{aligned}
$$

Evidently, this improves substantially the condition of LAD artery and provides an optimal resolution of this 'mother'-'daughter' configuration but at the 'cost' of one more revascularization.

\subsection{Example \#3 - A complex 4-artery configuration (Figure 3) - resolved by the formulas of Section 2.3}

3.3.1. The coronary configuration of Figure 3:(1) LMCA -

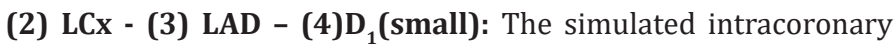
pressures for this MVD case are the following (in units of $\mathrm{mmHg}$ ):

$P_{a}=105 ; P_{p}=92 ; P_{2 d}=82 ; P_{3 d}=80 ; P_{4 d}=70$

Present status of the arteries (see formulas (18)):

$\operatorname{FFR}^{\text {true }}(1)=0.86 ; F^{\text {true }}(2)=0.89 ; F^{\text {true }}(3)=0.87 ; F^{\text {true }}(4)=0.88$

Note that FFR ${ }^{\text {true }}$ of an artery is equal to the maximal FFR ${ }^{\text {real }}$ obtained if most favorable conditions are created. Present real condition of the arteries (see formulas (17)):

$\operatorname{FFR}^{\text {real }}(1)=0.77 ; \mathrm{FFR}^{\text {real }}(2)=0.78 ; \mathrm{FFR}^{\text {real }}(3)=0.76 ; \mathrm{FFR}^{\text {real }}(4)=0.67$

By the treatment criteria of the hyperemic FFR method chosen to be followed in this article, the condition of the 4-artery configuration of (Figure 3) is obviously unacceptable (one of the FFR $^{\text {real }}$ values here is below the cut-off value of 0.75 whereas others barely exceed it). The PCI practitioner therefore needs to 
explore firstly the various revascularization options of the sizable arteries (that will be potentially also beneficial to the $\mathrm{D}_{1}$ artery) the outcomes of which can be predicted by formulas (A47) of Appendix A prior to possible implementation.

\subsubsection{Revascularization Options}

\subsubsection{Revascularization option a:}

From a glance at the present FFR ${ }^{\text {true }}$ values of the arteries it seems that revascularization of Artery 3 (LAD) (Figure 3) could improve substantially its condition, as well as the condition of Artery $4\left(D_{1}\right)$ and possibly of other arteries too. LAD artery has the lowest FFR $^{\text {true }}$ of all the sizable arteries of the configuration so elevating its FFR ${ }^{\text {true }}$ theoretically up to 1.00 (in practice it is somewhat less) could be most beneficial. In the optional revascularization of Artery 3 (LAD) therefore the FFR ${ }^{\text {true }}$ values of the other arteries remain at their present values while $\mathrm{FFR}^{\text {true }}(3)$ changes to 1.00 :

$\operatorname{FFR}^{\text {true }}(1)=0.86 ; F^{\text {true }}(2)=0.89 ; \operatorname{FFR}^{\text {true }}(3)=1.00 ; \operatorname{FFR}^{\text {true }}(4)=0.88$

Using these values in formulas (A47) of Appendix A, the postrevascularization condition of the sizable arteries can be predicted:

$\operatorname{FFR}^{\text {real }}(1)=0.82 ; \operatorname{FFR}^{\text {real }}(2)=0.77 ; \operatorname{FFR}^{\text {real }}(3)=0.87$

As expected, such a revascularization results in a substantial improvement of the condition of Artery 3 (LAD). Also, the condition of Artery 2 ( $\mathrm{LCx}$ ) remains the same (no change in its FFR $^{\text {real }}$ ) which is understandable since the blood flows in LAD and LCx are competing. The revascularization improves also the condition of Artery 1 (LMCA) because of the reduction in resistance to the outflow from LMCA. Let's turn now to Artery $4\left(D_{1}\right)$ and calculate its anticipated distal pressure $\mathrm{P}_{4 \mathrm{~d}}$ following the optional revascularization described here. Using formulas (19) one has (in units of $\mathrm{mmHg}$ ):

$$
\mathrm{P}_{4 \mathrm{~d}}=80
$$

This yields the condition of Artery $4\left(D_{1}\right)$ by formulas (20):

$$
\operatorname{FFR}^{\text {real }}(4)=0.76
$$

Which is a substantial improvement compared to its present condition but still barely acceptable. Note that FFR ${ }^{\text {true }}(4)$ does not change since it is an intrinsic property of $\mathrm{D}_{1}$ which has not been revascularized and it remains $\mathrm{FFR}^{\text {true }}(4)=0.88$.

If now artery $\mathrm{D}_{1}$ is revascularized, one has:

$\operatorname{FFR}^{\text {true }}(4)=1.00$

The intracoronary distal pressure is

$P_{4 d}=91$ (by formulas (19)) which yields

$\operatorname{FFR}^{\text {real }}(4)=0.87$

\subsubsection{Revascularization option b:}

Despite the potential improvement of the condition of several arteries, the downside of Revascularization option a is that the full potential of LMCA would not have been realized. The maximal FFR $^{\text {real }}$ of LMCA is equal to its FFR ${ }^{\text {true }}$ and this would not have been achieved. In the present revascularization option, the outcome of revascularizing both Artery 2 (LCx) and Artery 3 (LAD) will be therefore predicted. As the resistance to the outflow from LMCA would be drastically reduced, it is expected that such a revascularization would result in a substantial improvement of the condition of LMCA. Naturally, a substantial improvement would be also expected in the condition of arteries LCx and LAD, compared to their present condition. However, no significant improvement of the condition of $D_{1}$ beyond the improvement that could have been obtained in Revascularization option a) is expected.

In the present optional revascularization, the $\mathrm{FFR}^{\text {true }}$ values of the sizable arteries are the following:

$$
\operatorname{FFR}^{\text {true }}(1)=0.86 ; \mathrm{FFR}^{\text {true }}(2)=1.00 ; \mathrm{FFR}^{\text {true }}(3)=1.00
$$

The post-revascularization condition of the sizable arteries can be predicted from formulas (A47):

$\operatorname{FFR}^{\text {real }}(1)=0.86 ; \mathrm{FFR}^{\text {real }}(2)=0.86 ; \mathrm{FFR}^{\text {real }}(3)=0.86$

As expected, if this revascularization option had been realized, there would have been a substantial improvement in the condition of all 3 sizable arteries (compared to their present condition) and the full present potential of LMCA would have been realized.

Turning now to Artery $4\left(\mathrm{D}_{1}\right)$ one should take it into account that in the present revascularization option $\mathrm{D}_{1}$ artery is not involved directly therefore there is no change in its FFR ${ }^{\text {true }}$ and it remains

$\operatorname{FFR}^{\text {true }}(4)=0.88$

The post-revascularization value of $\mathrm{P}_{4 \mathrm{~d}}$ can be calculated (in units of mmHg) from formulas (19):

$\mathrm{P}_{4 \mathrm{~d}}=79$

$\mathrm{FFR}^{\text {real }}(4)$ can now be calculated from formulas (20):

$\operatorname{FFR}^{\text {real }}(4)=0.75$

As expected, the condition of Artery $4\left(D_{1}\right)$, should one carry out the present revascularization option, would be about the same as in Revascularization option a) which renders the condition of $\mathrm{D}_{1}$ barely acceptable.

If now artery $\mathrm{D}_{1}$ is revascularized, one has

$\mathrm{FFR}^{\text {true }}(4)=1.00$ and the distal intracoronary pressure is

$\mathrm{P}_{4 \mathrm{~d}}=90$ (by formulas (19)) which yields

$\mathrm{FFR}^{\text {real }}(4)=0.86$

This renders artery $\mathrm{D}_{1}$ quite acceptable.

\subsubsection{Conclusions from predicted outcomes of revascular- ization options}

Since a CABG operation is usually the way for revascularizing LMCA, this is not to be taken lightly and such an option is therefore avoided here. Option b) yields better results than option a) regarding the 3 sizable arteries. The two revascularization options improve substantially the condition of Artery $4\left(\mathrm{D}_{1}\right)$ compared to 
its present condition. Deplorably the improved condition of $D_{1}$ is barely acceptable because of its low FFR ${ }^{\text {real }}$, therefore stenting $\mathrm{D}_{1}$ would be beneficial, as shown here, if it is accessible geometrically (angle and diameter) and if it is functionally justifiable (namely if it is not too tiny to be hemodynamically of interest).

To conclude, under the circumstances, revascularization option b) (preferably combined with revascularization of $\mathrm{D}_{1}$ ) provides the optimal resolution of the stenotic 4-artery coronary configuration of (Figure 3).

\section{Discussion}

\subsection{Single-artery FFR-oriented methods}

About 25 years ago the emergence of the single-artery FFR method marked the dawn of a new era in the field of coronary stenosis severity assessment [1]. In contrast to the traditional visual and essentially subjective and qualitative stenosis severity assessment by the morphological appearance of the stenosis, FFRoriented methods are based on quantitative assessment of the functional performance of the stenotic artery. The 3 FFR-oriented single-artery methods, resting $\mathrm{P}_{\mathrm{d}} / \mathrm{P}_{\mathrm{a}}$ ratio, induced-hyperemia FFR and free-wave iFR are all based on intracoronary pressure measurements under very specific conditions that maintain a linearity relationship between blood flow and pressure drop. Because of the different techniques involved in the 3 FFR-oriented methods, each method has its specific FFR threshold value and its FFR 'grey range' of treatment-decision uncertainty. A typical threshold usually employed with the resting $\mathrm{P}_{\mathrm{d}} / \mathrm{P}_{\mathrm{a}}$ method is about 0.88 with a 'grey range' of about $0.88 \leq \mathrm{P}_{\mathrm{d}} / \mathrm{P}_{\mathrm{a}} \leq 0.95$ [16]. In the free wave iFR method, the threshold value is about 0.86 with a 'grey range' of about $0.86 \leq \mathrm{iFR} \leq 0.93$ [17]. As indicated already in this article, a threshold value in the hyperemic FFR method is about 0.75 with a 'grey range' of about $0.75 \leq$ FFR $\leq 0.85$ [5].

The 3 single-artery FFR-oriented coronary stenosis severity assessment methods are well designed to handle SVD cases, both simple cases with a single localized stenosis and more complex cases that involve serial stenoses within the same artery [18]. The phenomenon of collaterals has been also addressed by some of these methods [4]. However, the reality of the epicardial arterial network is in most cases much more complicated than that of a single artery. Initial attempts to study the effect of downstream stenoses (in LAD and LCx arteries) on unprotected LMCA by a single-artery approach have not yielded a rigorous resolution of stenotic unprotected LMCA-LCX-LAD configurations on a case to case basis, rather general quantitative results $[9,10]$.

\subsection{Multi-artery FFR method}

The specific conditions under which the intracoronary pressure measurements are carried out in the single-artery FFRoriented methods maintain a linearity relationship between blood flow through a stenosis and the associated pressure drop over the stenosis. The epicardial arterial network is analogous to an electric interconnected network of conductors and resistors which implies that the rules that govern the relationship between the epicardial intracoronary pressures and blood flow are analogous to Kirchhoff's laws of direct-current circuits. These analogous rules combined with appropriate mathematical transformations constitute the multi-artery FFR method which can practically extend each of the 3 single-artery coronary stenosis severity assessment methods to the multi-artery domain. This extension does not violate the basic experimental techniques nor the treatment-decision criteria of any of the single-artery methods. However, the virtual transition from the single artery 'stand-alone' position to the multi-artery scenario is in principle associated with a substantial change of circumstances. Therefore, in the multi-artery scenario these criteria no longer apply to FFR ${ }^{\text {true }}$ of a stenotic artery, rather to its actual FFR, namely to its FFR ${ }^{\text {real }}$ that reflects the current condition of an artery. In a multi-artery coronary configuration, the FFR ${ }^{\text {true }}$ of a stenotic artery assumes a new role, it is potentially the maximal possible $\mathrm{FFR}^{\text {real }}$ of the artery, $\mathrm{FFR}^{\text {real }} \leq \mathrm{FFR}^{\text {true }}$. This current maximal potential of $\mathrm{FFR}^{\text {real }}$ is fully realized if the aortic pressure is maintained all the way to the beginning of the stenotic artery. The potential is further increased if the stenotic artery itself is revascularized and its FFR ${ }^{\text {true }}$ is turned into nearly 1.00. The most important aspect of the superiority of the multi-artery FFR method over the single-artery FFR-oriented methods is the inclusion of inter-arterial interactions.

The capability of handling stenotic coronary multi-artery configurations of interacting arteries was not available to the designers of the FAME study [8] in 2009. They chose to get around this obstacle by excluding cases of diseased LMCA from the FAME study. This exclusion rendered the LCx and LAD arteries independent of each other and reduced the seemingly MVD cases of the study practically to SVD cases and they were indeed treated as such in that study. The multi-artery FFR method emerged in 2015 [11] and the mathematical formulas for resolving the stenotic LMCA-LCX-LAD configuration (and other 3-artery configurations of sizable arteries) were finalized in 2017 [13]. Nevertheless, there are still researchers that apply the 'exclusion principle' to cases of diseased LMCA in their statistical studies even in 2020 [19] thus losing unnecessarily invaluable statistical data that would have potentially affected the results of those studies. What may have been understandable under the circumstances in 2009 should not be acceptable in 2020 .

\subsection{Current obstacles and future perspectives}

Theoretically the multi-artery FFR method seems superior to the single-artery FFR-oriented methods by the mere inclusion of inter-arterial interactions. However, it should be noted that the analogy between the epicardial arterial network and an electric interconnected network of conductors and resistors is not complete. Resistors are discrete components whereas stenoses are not always localized. LMCA stands out as an example. The plaque in LMCA is often diffusely distributed and in most cases protrudes also partly into the neighboring LCx and LAD arteries [20]. Under such circumstances, when the boundaries of a diffuse plaque are not well-defined, the accuracy of the FFR ${ }^{\text {true }}$ and FFR ${ }^{\text {real }}$ values obtained 
by the multi-artery FFR method is reduced. This can be alleviated to some extent by measuring separately the proximal intracoronary pressure of LCx artery and the proximal intracoronary pressure of LAD artery and regarding their arithmetic average as the common proximal intracoronary pressure of arteries LCx and LAD [15] (Pp in Figure 1).

It should be noted that it would be beneficial to have the quality of revascularizations of stenotic arteries checked postrevascularization by the PCI practitioner in limiting cases (by the same kind of intracoronary pressure measurements that preceded the revascularizations) so that operational conclusions can be drawn if necessary. In the numerical examples in this article (3. Results - Numerical Simulation Examples) it has been assumed that the FFR ${ }^{\text {true }}$ value of a revascularized artery is 1.00 but, it might be less, as indicated already. In this context note that possible different results of the FFR $^{\text {real }}$ values of the arteries due to such a variation of FFR ${ }^{\text {true }}$ can be observed in Example 3 when outcomes of Revascularization option a) (with $\mathrm{FFR}^{\text {true }}(2)=0.89$ ) and Revascularization option b) (with $\operatorname{FFR}^{\text {true }}(2)=1.00$ ) are considered. On the practical level, the multi-artery FFR method is very convenient. Firstly, the PCI practitioner can readily identify to what category belongs the arterial scenario encountered in the catheterization laboratory. Then the practitioner turns to the right pressure-resistance-flow scheme and measures the appropriate intracoronary pressures. This experimental data is then used with the relevant formulas of paragraph 2. Methodology - The Basic Essentials in order to obtain the current values of FFR ${ }^{\text {true }}$ and $\mathrm{FFR}^{\text {real }}$ of each artery of the arterial scenario. At this point it is important not to be led astray by the values of FFR ${ }^{\text {true }}$ and FFR ${ }^{\text {real }}$ of an artery. Arteries with a reasonable FFR $^{\text {true }}$ may end up with a very low and unacceptable FFR ${ }^{\text {real }}$. This should not come as a surprise because unlike in the SVD case, the presence of each additional stenotic artery in the configuration impedes further the blood flow through the multi-artery system.

It is important to comprehend the meaning of $\mathrm{FFR}^{\text {true }}$ and $\mathrm{FFR}^{\text {real }}$. The initial $\mathrm{FFR}^{\text {real }}$ (prior to possible revascularizations) reflects the current condition of an artery and therefore regardless of the FFR-oriented method that is being used, hyperemic FFR or instantaneous free-wave iFR or resting $\mathrm{P}_{d} / \mathrm{P}_{\mathrm{a}}$, the treatmentdecision criteria should be applied to $\mathrm{FFR}^{\text {real }}$, rather than to $\mathrm{FFR}^{\text {true }}$ If the value of FFR real is less than the FFR threshold value associated with the method that is being used, the condition of the artery is unacceptable. At this point, it would be beneficial to take also a good look at the numerical value of FFR ${ }^{\text {true }}$ obtained for each artery since this may point the way to devising the most appropriate revascularization strategy on the way to optimal resolution of the stenotic coronary configuration. FFR $^{\text {true }}$ indicates potential, not condition. It is the upper bound of possible FFR ${ }^{\text {real }}$ for the artery that can be obtained by revascularization of other arteries. If the value of FFR ${ }^{\text {true }}$ of an artery is too low, there is no alternative but to revascularize the artery itself (turning its FFR true to nearly 1.00), provided that it is technically feasible and that the artery is hemodynamically sufficiently important to the coronary blood circulation. Such a revascularization provides direct relief to the artery or enables potential relief through optional revascularization of other arteries.

There are two downsides of the multi-artery FFR method that involve strategy. Firstly, the method does not (and actually cannot) consider the exact location of stenoses. Assuming a same FFR ${ }^{\text {real }}$, a stenosis in the proximal section of an artery potentially puts at risk more downstream side branches than a stenosis in the distal section of an artery. Such a matter can be taken into consideration only by a PCI practitioner who can weigh the importance of such a state of a configuration. Secondly, when the PCI practitioner is confronted with an unfavorable numerical value of FFR ${ }^{\text {real }}$ of an artery then if FFR $^{\text {real }}$ is below the lower end of the 'grey zone' of the FFR treatment decision range then it is undisputedly not acceptable. However, if FFR $^{\text {real }}$ is within the 'grey zone' or slightly above it, it is up to the PCI practitioner to decide what can be regarded 'safe' for the patient under consideration, regarding also future prospects given the patient's way of life, and if revascularization would be a clinically justifiable 'price' for it. Although the multi-artery FFR method provides the PCI practitioner with precise data about the status of a configuration (namely FFR ${ }^{\text {real }}$ and FFR ${ }^{\text {true }}$ of each artery), in order to devise a strategy of handling a complex stenotic coronary configuration, professional experience and careful judgement on the part of the PCI practitioner are virtually irreplaceable.

In some cases, the final stages of the procedure, predicting the outcomes of optional revascularizations, are obvious to the experienced PCI practitioner. However, should the practitioner nevertheless choose to continue following closely the multi-artery FFR method in the footsteps given in this article (Appendix A), the appropriate formulas are relatively simple and can be used by the PCI practitioner in real time or incorporated for convenience into a software application module. Note that such a software application module should enable constant scrutiny from the PCI practitioner who inputs the data (measured intracoronary pressures, FFR $^{\text {true }}$ values, optional $\mathrm{FFR}^{\text {true }}$ values, as appropriate) and the output data (FFR ${ }^{\text {true }}$ and FFR $^{\text {real }}$ values) should be presented in raw form for direct evaluation by the practitioner. The practitioner can use his/ her past experience from SVD cases in order to visually estimate FFR $^{\text {true }}$ of arteries and so rudimentarily verify the validity of the output data from the software application module to be assured that the output data is flawless and very likely reflects reality (note however that FFR $^{\text {real }}$ of an artery may be substantially different from estimated FFR ${ }^{\text {true }}$ and therefore cannot be used for verification purposes).

It should be noted that the intracoronary pressure values used in the numerical simulations for the demonstration of the capabilities of the multi-artery FFR method [11-14] have been all taken from the experimental intracoronary pressure range encountered in the catheterization lab. The obvious current advantage of the multiartery FFR method is therefore the possible smooth transition 
from the use of simulated intracoronary pressures to the use of real intracoronary pressure data obtained in the catheterization lab.

\section{Conclusion}

The current FFR-oriented methods (resting $\mathrm{P}_{\mathrm{d}} / \mathrm{P}_{\mathrm{a}}$, wave-free iFR and hyperemic FFR) are single-artery methods that cannot resolve stenotic multi-artery configurations in which inter arterial interactions take place. The multi-artery FFR method extends these methods to the multi-artery domain without imposing any changes in the experimental technique of any of these methods nor in the treatment decision criteria (FFR threshold value and FFR 'grey range') of any of them. The multi-artery FFR method is not intended to constitute a substitute for any of the current single-artery FFRoriented methods. It however provides these methods with the necessary capabilities for resolving stenotic multi-artery coronary configurations.

\section{Acknowledgement}

None.

\section{Conflict of Interest}

No conflict of interest.

\section{References}

1. Sarno G, Lagerqvist B, Frobert O, Nilsson J, Olivecrona G, et al. (2012) Lower risk of stent thrombosis and restenosis with unrestricted use of 'new-generation' drug-eluting stents: a report from the nationwide Swedish Coronary Angiography and Angioplasty Registry (SCAAR). Eur Heart J 33: 606-613.

2. Dangas GD, Claessen BE, Caixeta A, Sanidas EA, Mintz GS, et al. (2010) In-Stent Restenosis in the Drug-Eluting Stent Era. J Am Coll Cardiol 56: 1897-1907.

3. Iakovou I, Schmidt T, Bonizzoni E, Ge L, Sangiorgi GM, et al. (2005) Incidence, Predictors, and Outcome of Trombosis After Successful Implantation of Drug-Eluting Stents. JAMA 293: 2126-2130.

4. Pijls NH, van Son JA, Kirkeeide RL, De Bruyne B, Gould KL (1993) Experimental basis of determining maximum coronary, myocardial, and collateral blood flow by pressure measurements for assessing functional stenosis severity before and after percutaneous transluminal coronary angioplasty. Circulation 87: 1354-1367.

5. Petraco R, Sen S, Nijjer S, Echavarria-Pinto M, Escaned J, et al. (2013) Fractional Flow Reserve-Guided Revascularization - Practical Implications of a Diagnostic Gray Zone and Measurement Variability on Clinical Decisions. JACC Cardiovasc Interv 6: 222-225.

6. Sen S, Escaned J, Malik IS, Mikhail GW, Foale RA, et al. (2012) Development and validation of a new adenosine-independent index of stenosis severity from coronary wave-intensity analysis: results of the ADVISE (Adenosine Vasodilator Independent Stenosis Evaluation) study. J Am Coll Cardiol 59: 1392-1402.
7. de Waard GA, Danad I, Petraco R, Driessen RS, Raijmakers PG, et al. (2018) Fractional flow reserve, instantaneous wave-free ratio, and resting Pd/Pa compared with [150] H2O positron emission tomography myocardial perfusion imaging: a PACIFIC trial sub-study. Eur Heart J 39: 4072-4081.

8. Tonino PAL, De Bruyne B, Pijls NHJ, Siebert U, Ikeno F, et al. (2009) Fractional Flow Reserve versus angiography for guiding Percutaneous Coronary Intervention. N Engl J Med 360: 213-224.

9. Fearon WF, Yong AS, Lenders G, Toth GG, Dao C, et al. (2015) The impact of downstream coronary stenosis on fractional flow reserve assessment of intermediate left main coronary artery disease: human validation. JACC Cardiovasc Interv 8: 398-403.

10. Daniels DV, van't Veer M, Pijls NHJ, van der Horst A, Yong AS, et al. (2012) The Impact of Downstream Coronary Stenoses on Fractional Flow Reserve Assessment of Intermediate Left Main Disease. JACC Cardiovasc Interv 5: 1021-1025.

11. Yaeger IA (2016) A multi-artery Fractional Flow Reserve (FFR) approach for handling coronary stenosis-stenosis interaction in the multi-vessel disease (MVD) arena. Int J Cardiol 203: 807-815

12. Yaeger IA (2015) Practicability of Multi-Artery Fractional Flow Reserve (FFR) Method in the Assessment of Some Stenotic Coronary Artery Configurations in Percutaneous Coronary Intervention (PCI) Procedures. Interv Cardiol J 2(3): 31.

13. Yaeger IA (2017) Resolution of All-stenotic 3-Artery Configurations in Percutaneous Coronary Intervention (PCI) Procedures by the Multiartery Fractional Flow Reserve (FFR)Method. Cardiovasc Investig 1(1):

14.Yaeger IA (2018) Resolution of coronary 'mother'-'daughter' configurations by multi-artery FFR method - a comparison with basic FFR method. Interv Cardiol 10: 65-71.

15. Yaeger IA (2019) Strategies of Handling a Stenotic Unprotected Left Main Coronary Artery by Multi-Artery Fractional Flow Reserve Method. On J Cardiovas Res 1(4): 1-7.

16. Ammar KA, Kazmi SS, Ahmad MN, Ahmad MM, Riaz A, et al. (2016) Use of Resting Non-hyperemic Indices for Avoidance of Fractional Flow Reserve Measurement: The Goal of 100\% Accuracy. I Invasive Cardiol 28: $265-270$.

17. Petraco R, Escaned J, Sen S, Nijjer S, Asrress KN, et al. (2013) Classification performance of instantaneous wave-free ratio (iFR) and fractional flow reserve in a clinical population of intermediate coronary stenoses: results of the ADVISE registry. EuroIntervention 9: 91-101.

18. De Bruyne B, Pijls NHJ, Heyndrickx GR, Hodeige D, Kirkeeide R, et al (2000) Pressure-Derived Fractional Flow Reserve to Assess Serial Epicardial Stenoses Theoretical Basis and Animal Validation. Circulation 101: 1840-1847.

19. Wijntjens GWM, van de Hoef TP, Meuwissen M, Echavarría-Pinto M Murai T, et al. (2020) Prognostic implications of resting distal coronaryto-aortic pressure ratio compared with fractional flow reserve: a 10year follow-up study after deferral of revascularization. Neth Heart J 28 : 96-103.

20. Oviedo C, Maehara A, Mintz GS, Araki H, Choi SY, et al. (2010) Intravascular Ultrasound Classification of Plaque Distribution in Left Main Coronary Artery Bifurcations - Where Is the Plaque Really Located. Circ Cardiovasc Interv 3: 105-112. 


\section{The Multi-Artery FFR Method - Concise Methodology}

\section{First appearance and the basic mathematical approach}

The first appearance online of the multi-artery FFR method at the end of 2015 [1] was in the wake of attempts by some researchers to study the effect of downstream stenoses on the LMCA by the basic hyperemic FFR method [2,3]. In order to avoid technical artifacts, only cases with one stenotic downstream artery (either LAD or LCx) were considered in those attempts and the LMCA distal intracoronary pressure $\mathrm{P}_{\mathrm{p}}$ was measured in the non-stenotic downstream artery. The configuration under consideration could be denoted [stenotic LMCA] - [nonstenotic LCx] - [stenotic LAD]. In those studies, the single-artery hyperemic FFR method was employed and only general quantitative results were obtained. Optimal resolution of this specific configuration on a case to case basis was eventually obtained numerically only by the multi-artery FFR method using a tabular approach [1]. The tabular approach consists essentially of 3 tables in which the elements of each table are the FFR ${ }^{\text {real }}$ values of one of the 3 arteries of the configuration as a function of FFR ${ }^{\text {true }}$ values of LMCA and FFR ${ }^{\text {true }}$ values of LAD artery. The rows of each table were labeled by the FFR ${ }^{\text {true }}$ values of stenotic LMCA whereas the columns were labeled by the $\mathrm{FFR}^{\text {true }}$ values of stenotic LAD artery.

Due to the very specific characteristics of the above scenario, in which only one of the two downstream arteries was stenotic, the resolution of the configuration could be reduced to a 2 -variable problem and the tabular approach was appropriate [1]. The tabular approach however will not be described here because it involves numerical interpolation of table elements that might be inconvenient in real-time work and because much more convenient algebraic resolution techniques of stenotic configurations will be presented in this appendix.

Scenarios in which 3 interconnected coronary arteries are stenotic are not uncommon. A typical pressure-resistance-flow scheme of a 3 -artery configuration is presented in (Figure 1). Artery 1, which can be regarded functionally as a conductance artery, bifurcates into arteries 2 and 3. The latter two arteries are end arteries that lead through the microvasculature to different regions of the myocardium. The negligible pressure of the venous bed, $\mathrm{P}_{\mathrm{v}}$, is taken to be zero and arteries 2 and 3 (that both lead therefore to zero pressure) can be regarded as being effectively in a parallel connection with each other. They are preceded by artery 1 which is connected to them in series. The scheme of these 3 arteries in (Figure 1) will be used to study MVD scenarios. It should be noted however that other stenotic 3-artery and 2-artery equivalent configurations of coronary arteries can be also resolved by employing a scheme identical to the one of (Figure 1), as will be indicated later. Using the analogous electricity rules for obtaining the equivalent resistance of resistors connected in parallel and resistors connected in series, the magnitude of the blood flow through each artery can be calculated and the expressions for the mean intracoronary pressures $\mathrm{P}_{\mathrm{p}}, \mathrm{P}_{2 \mathrm{~d}}$ and $\mathrm{P}_{3 \mathrm{~d}}$ at the appropriate locations (Figure 1) can be obtained:

$$
\begin{aligned}
& \mathrm{P}_{\mathrm{p}}=\mathrm{P}_{\mathrm{a}} /\left\{\left(\mathrm{R}_{1 \mathrm{~s}} / \mathrm{R}_{3 \mathrm{mv}}\right) /\left[1+\left(\mathrm{R}_{3 \mathrm{~s}} / \mathrm{R}_{3 \mathrm{mv}}\right)\right]+\right. \\
& \left.\left(\mathrm{R}_{1 \mathrm{~s}} / \mathrm{R}_{2 \mathrm{mv}}\right) /\left[1+\left(\mathrm{R}_{2 s} / \mathrm{R}_{2 \mathrm{mv}}\right)\right]+1\right\} \\
& \mathrm{P}_{2 \mathrm{~d}}=\mathrm{P}_{\mathrm{a}} /\left\{\left(\mathrm{R}_{1 s} / \mathrm{R}_{2 \mathrm{mv}}\right)+\left(\mathrm{R}_{2 \mathrm{~s}} / \mathrm{R}_{2 \mathrm{mv}}\right)+1+\right. \\
& {\left[\left(\mathrm{R}_{1 \mathrm{~s}} / \mathrm{R}_{3 \mathrm{mv}}\right) /\left[\left(\mathrm{R}_{3 \mathrm{~s}} / \mathrm{R}_{3 \mathrm{mv}}\right)+1\right] \cdot\left[\left(\mathrm{R}_{2 \mathrm{~s}} / \mathrm{R}_{2 \mathrm{mv}}\right)+1\right]\right\}} \\
& \mathrm{P}_{3 \mathrm{~d}}=\mathrm{P}_{\mathrm{a}} /\left\{\left(\mathrm{R}_{1 s} / \mathrm{R}_{3 \mathrm{mv}}\right)+\left(\mathrm{R}_{3 \mathrm{~s}} / \mathrm{R}_{3 \mathrm{mv}}\right)+1+\right. \\
& {\left[\left(\mathrm{R}_{1 \mathrm{~s}} / \mathrm{R}_{2 \mathrm{mv}}\right) /\left[\left(\mathrm{R}_{2 \mathrm{~s}} / \mathrm{R}_{2 \mathrm{mv}}\right)+1\right] \cdot\left[\left(\mathrm{R}_{3 \mathrm{~s}} / \mathrm{R}_{3 \mathrm{mv}}\right)+1\right]\right\}}
\end{aligned}
$$

In the various resistances $\mathrm{R}_{\mathrm{j} \mathrm{k}}$ in these expressions the first index indicates the artery number $\mathrm{j}=1,2,3$ while the second index $\mathrm{k}$ indicates the kind of resistance, stenotic (s) or microvascular (mv).

It can be seen that the above expressions are actually functions of the following 4 variables: $\mathrm{R}_{1 \mathrm{~s}} / \mathrm{R}_{2 \mathrm{mv}} \mathrm{R}_{1 \mathrm{~s}} / \mathrm{R}_{3 \mathrm{mv}}, \mathrm{R}_{2 \mathrm{~s}} / \mathrm{R}_{2 \mathrm{mv}}$ and $\mathrm{R}_{3 \mathrm{~s}} / \mathrm{R}_{3 \mathrm{mv}}$.

The $\mathrm{P}_{\mathrm{p}}, \mathrm{P}_{2 \mathrm{~d}}$ and $\mathrm{P}_{3 \mathrm{~d}}$ expressions can be eventually written as functions of FFR ${ }^{\text {true }}(1), F^{\text {true }}(2), F^{\text {true }}(3)$ and $\delta$ which is defined as following:

$$
\delta=\mathrm{R}_{3 \mathrm{mv}} / \mathrm{R}_{2 \mathrm{mv}}
$$

The dependence of the intracoronary pressures on the $\mathrm{FFR}^{\text {true }}$ of the arteries stems from the following familiar relationships:

$$
\begin{aligned}
& \operatorname{FFR}^{\text {true }}(1)=1 /\left[\left(\mathrm{R}_{1 \mathrm{~s}} / \mathrm{R}_{2 \mathrm{mv}}\right)+\left(\mathrm{R}_{1 \mathrm{~s}} / \mathrm{R}_{3 \mathrm{mv}}\right)+1\right] \\
& \operatorname{FFR}^{\text {true }}(2)=1 /\left[1+\left(\mathrm{R}_{2 \mathrm{~s}} / \mathrm{R}_{2 \mathrm{mv}}\right)\right] \\
& \operatorname{FFR}^{\text {true }}(3)=1 /\left[1+\left(\mathrm{R}_{3 \mathrm{~s}} / \mathrm{R}_{3 \mathrm{mv}}\right)\right]
\end{aligned}
$$

The basic relationships presented in this section will be incorporated into the formulas for the resolution of the coronary scenarios that belong to the relevant categories of arterial configurations described in the following sections.

\section{Generalized formulas for the resolution of sten- otic 3-artery coronary configurations}

In this section the multi-artery FFR method is generalized to include the capability to resolve stenotic 3-artery coronary configurations of sizable arteries (Figure 1) in which all the arteries are potentially stenotic and also configurations in which one of the arteries is a small side branch (Figure 2) as well as a considerable number of other equivalent configurations. In such cases the intracoronary pressures are in principle dependent on 4 variables:

$\mathrm{FFR}^{\text {true }}(1), \mathrm{FFR}^{\text {true }}(2), \mathrm{FFR}^{\text {true }}(3)$ and $\delta$. 
The importance of this dependence may seem purely theoretical since the current intracoronary pressures are usually obtained simply by measurement, not by calculations. However, should the PCI practitioner desire to predict the outcome of optional revascularizations on the way to achieve optimal resolution of the stenotic configuration, the anticipated intracoronary pressures can be only calculated from the FFR $^{\text {true }}$ input data used in those revascularizations. The importance of these pressures stems from the possibility that some of them might be post-revascularization driving pressures of small side branches originating from sizable arteries (see Section 2 here and Example \#3 in Section 3.3 of the article).

The anticipated intracoronary pressures are expressed in terms of $\mathrm{FFR}^{\text {true }}(1), \mathrm{FFR}^{\text {true }}(2), \mathrm{FFR}^{\text {true }}(3)$ and $\delta$ as following

$$
\begin{aligned}
& \mathrm{P}_{\mathrm{p}}=\mathrm{P}_{\mathrm{a}} \cdot \mathrm{FFR}^{\text {true }}(1) /\left\{\{ [ 1 / ( 1 + \delta ) ] \cdot [ 1 - \mathrm { FFR } ^ { \text { true } } ( 1 ) ] \} \cdot \left[\delta \cdot \mathrm{FFR}^{\text {true }}(2)+\right.\right. \\
& \left.\left.\mathrm{FFR}^{\text {true }}(3)\right]+\mathrm{FFR}^{\text {true }}(1)\right\}
\end{aligned}
$$

$$
\begin{aligned}
& \mathrm{P}_{2 \mathrm{~d}}=\mathrm{P}_{\mathrm{a}} \cdot \mathrm{FFR}^{\text {true }}(2) \cdot \mathrm{FFR}^{\text {true }}(1) /\left\{\left\{[1 /(1+\delta)] \cdot\left[1-\mathrm{FFR}^{\text {true }}(1)\right]\right\}\right. \\
& \left.\left[\delta \cdot \mathrm{FR}^{\text {true }}(2)+\mathrm{FFR}^{\text {true }}(3)\right]+\mathrm{FFR}^{\text {true }}(1)\right\}
\end{aligned} \cdot
$$

$$
\mathrm{P}_{3 \mathrm{~d}}=\mathrm{P}_{\mathrm{a}} \cdot \mathrm{FFR}^{\text {true }}(3) \cdot \mathrm{FFR}^{\text {true }}(1) /\left\{\left\{[1 /(1+\delta)] \cdot\left[1-\mathrm{FFR}^{\text {true }}(1)\right]\right\} \cdot\right.
$$$$
\left.\left[\delta \cdot \mathrm{FFR}^{\text {true }}(2)+\mathrm{FFR}^{\text {true }}(3)\right]+\mathrm{FFR}^{\text {true }}(1)\right\}
$$

Note that all these 3 expressions have a common denominator which facilitates calculations.

The dependence of the intracoronary pressures on $\delta$ deserves a special attention. The microvascular resistance of a coronary artery is closely related to its morphology [4]. This seems to suggest an undesirable dependence of the formulas of the multiartery FFR method on the geometry of the arteries of the 3-artery configuration that may vary from person to person. Consequently, this matter was thoroughly looked into [4]. It turned out that in stenotic 3 artery configurations of sizable coronary arteries in typical scenarios within ordinary range of statistical variation of morphology (radii, rates of tapering and lengths) of coronary arteries [5] $\delta$ is in the range $0.6<\delta<1.7$ namely in the proximity of $\delta=1$, if the geometrical average of the endpoints of the $\delta$ range is considered $[4,6]$. Calculations show that $\mathrm{FFR}^{\text {real }}$ (as functions of $\delta$ ) for the individual arteries exhibit acceptable small variations of just $\Delta \mathrm{FFR}^{\text {real }}= \pm 0.02$ within this range of $\delta$ in the low and intermediate stenosis severity ranges of the arteries [4]. With regard to the 3 -artery configuration of sizable coronary arteries of (Figure 1 ), $\delta$ can be considered not as a variable, rather as a constant $\delta=1$ [6] and practically no $\delta$ correction terms are needed upon going from one patient to the next.

However, the value used for $\delta$ changes entirely if Artery 3 of the arterial configuration in (Figure 2) represents a small side branch while Artery 1 and Artery 2 remain sizable arteries. The blood flow through a small artery is lower than the flow through a sizable artery which implies also a lower number of arteriole-capillary complexes connected in parallel within the microvasculature [4]. This in turn implies a substantially greater microvascular resistance resulting in a relatively higher $\delta$, namely $1<<$. This condition is practically obtained within the formulas by mathematically letting $\delta$ tend to infinity, $\delta \rightarrow \infty[4,7]$. It will be shown however that contrary to what it may presently seem, the final formulas employed by the PCI practitioner in such cases are relatively simple.

The PCI practitioner can obtain the current values of FFR ${ }^{\text {true }}$ of each one of the arteries from the measured intracoronary pressures by using the following formulas $[4,6]$ :

$$
\begin{aligned}
& \operatorname{FFR}^{\text {true }}(1)=1 /\left\{\left[\left(\mathrm{P}_{\mathrm{a}}-\mathrm{P}_{\mathrm{p}}\right) \cdot(1+\delta)\right] /\left(\mathrm{P}_{3 \mathrm{~d}}+\delta \cdot \mathrm{P}_{2 \mathrm{~d}}\right)+1\right\} \\
& \operatorname{FFR}^{\text {true }}(2)=\mathrm{P}_{2 \mathrm{~d}} / \mathrm{P}_{\mathrm{p}} \\
& \operatorname{FFR}^{\text {true }}(3)=\mathrm{P}_{3 \mathrm{~d}} / \mathrm{P}_{\mathrm{p}}
\end{aligned}
$$

In the derivation of the formulas presented here, FFR ${ }^{\text {true }}$ of an artery can be regarded as an intrinsic property of the stenotic artery. It characterizes the stenotic artery and does not change unless the artery is revascularized, in which case FFR ${ }^{\text {true }}$ becomes nearly 1.00. The condition of an artery however is determined by the value of its FFR ${ }^{\text {real }}$. Current FFR ${ }^{\text {real }}$ of each artery can be obtained from the measured intracoronary pressures [6]:

$$
\begin{aligned}
& \operatorname{FFR}^{\text {real }}(1)=\left(\mathrm{P}_{3 \mathrm{~d}}+\delta \cdot \mathrm{P}_{2 \mathrm{~d}}\right) /\left[\mathrm{P}_{\mathrm{a}} \cdot(1+\delta)\right] \\
& \operatorname{FFR}^{\text {real }}(2)=\mathrm{P}_{2 \mathrm{~d}} / \mathrm{P}_{\mathrm{a}} \\
& \operatorname{FFR}^{\text {real }}(3)=\mathrm{P}_{3 \mathrm{~d}} / \mathrm{P}_{\mathrm{a}}
\end{aligned}
$$

When the PCI practitioner wants to predict the outcome of optional revascularizations of some of the configurational arteries, the $\mathrm{FFR}^{\text {real }}$ of the arteries need to be expressed in terms of $\mathrm{FFR}^{\text {true }}$ (1), $\operatorname{FFR}^{\text {true }}(2), \mathrm{FFR}^{\text {true }}(3)$ and $\delta[6]$ :

$$
\begin{aligned}
& \mathrm{FFR}^{\text {real }}(1)=\left\{\mathrm{FFR}^{\text {true }}(1) \cdot\left[\mathrm{FFR}^{\text {true }}(3)+\delta \cdot \mathrm{FFR}^{\text {true }}(2)\right]\right\} /\left\{\left[1-\mathrm{FFR}^{\text {true }}\right.\right. \\
& \left.(1)] \cdot\left[\delta \cdot \mathrm{FFR}^{\text {true }}(2)+\mathrm{FFR}^{\text {true }}(3)\right]+(1+\delta) \cdot \mathrm{FFR}^{\text {true }}(1)\right\}
\end{aligned}
$$

$\mathrm{FFR}^{\text {real }}(2)=\left\{\left[(1+\delta) \cdot \mathrm{FFR}^{\text {true }}(1) \cdot \mathrm{FFR}^{\text {true }}(2)\right]\right\} /\left\{\left[1-\mathrm{FFR}^{\text {true }}(1)\right] \cdot\right.$ $\left.\left[\delta \cdot F^{\text {true }}(2)+\mathrm{FFR}^{\text {true }}(3)\right]+(1+\delta) \cdot \mathrm{FFR}^{\text {true }}(1)\right\}$

$\mathrm{FFR}^{\text {real }}(3)=\left\{\left[(1+\delta) \cdot \mathrm{FFR}^{\text {true }}(1) \cdot \mathrm{FFR}^{\text {true }}(3)\right]\right\} /\left\{\left[1-\mathrm{FFR}^{\text {true }}(1)\right] \cdot\right.$ $\left.\left[\delta \cdot \mathrm{FFR}^{\text {true }}(2)+\mathrm{FFR}^{\text {true }}(3)\right]+(1+\delta) \cdot \mathrm{FFR}^{\text {true }}(1)\right\}$

Noticeably FFR ${ }^{\text {real }}(1)$, FFR ${ }^{\text {real }}(2)$ and FFR ${ }^{\text {real }}(3)$ have a common denominator in these expressions. This will prove useful for shortening the data processing time when optional revascularization outcomes are evaluated in PCI procedures. When using these expressions, FFR ${ }^{\text {true }}$ of an artery that is not revascularized is identical to its original value whereas FFR ${ }^{\text {true }}$ of a revascularized artery is set to $\mathrm{FFR}^{\text {true }}=1.00$ for calculation purposes. The $\mathrm{FFR}^{\text {real }}$ expressions (in terms of FFRtrue of the arteries) yield their condition should an optional revascularization take place.

\subsection{Resolution of Stenotic 3-Artery Coronary Configura- tions of Sizable Arteries}

In this section the scheme of (Figure 1) is employed for the representation of a 3-artery coronary configuration of sizable arteries that are all potentially stenotic. The most commonly encountered configuration of this kind in the PCI practice is the 3-artery configuration [LMCA]-[LCx]-[LAD] [6]. There are also 
other configurations for which the configuration of (Figure 1) can be regarded as an equivalent configuration and resolved accordingly, as will be described at the end of this section.

As indicated already in Section 2, in the resolution of stenotic 3 -artery coronary configurations of sizable arteries $\delta$ is set to $\delta=$ 1. In the presence of other stenotic arteries of the configuration that interact with each artery of the configuration, the value of FFR $^{\text {true }}$ no longer reflects the condition of an artery by which the way of treatment would have been determined if the artery had been in an independent stand-alone position. Although the condition of the artery is reflected by its FFR ${ }^{\text {real }}$, the FFR ${ }^{\text {true }}$ of the arteries are nevertheless very important because beside being an intrinsic property of the stenotic artery, FFR ${ }^{\text {true }}$ of the arteries are the auxiliary variables on which essential properties and features associated with the arteries depend.

When $\delta$ is set to $\delta=1$, expressions (A11) - (A13) for the FFR ${ }^{\text {true }}$ of the arteries assume the following forms:

$$
\begin{aligned}
& \operatorname{FFR}^{\text {true }}(1)=1 /\left\{\left[2 \cdot\left(\mathrm{P}_{\mathrm{a}}-\mathrm{P}_{\mathrm{p}}\right)\right] /\left(\mathrm{P}_{3 \mathrm{~d}}+\mathrm{P}_{2 \mathrm{~d}}\right)+1\right\} \\
& \operatorname{FFR}^{\text {true }}(2)=\mathrm{P}_{2 \mathrm{~d}} / \mathrm{P}_{\mathrm{p}} \\
& \operatorname{FFR}^{\text {true }}(3)=\mathrm{P}_{3 \mathrm{~d}} / \mathrm{P}_{\mathrm{p}}
\end{aligned}
$$

As indicated already, for the purpose of this analysis, FFR ${ }^{\text {true }}$ of an artery can be regarded as an intrinsic property of an artery. It remains constant, unless the artery is revascularized turning its FFR $^{\text {true }}$ to nearly 1.00

In search for the optimal resolution of the stenotic coronary configuration, the PCI practitioner explores the outcomes of optional revascularizations of some of the arteries of the configuration. The practitioner can also calculate the anticipated intracoronary pressures resulting from those revascularizations. The expressions (A8) - (A10) for the anticipated intracoronary pressures then assume the following forms:

$$
\begin{aligned}
& \mathrm{P}_{\mathrm{p}}=\mathrm{P}_{\mathrm{a}} \cdot \mathrm{FFR}^{\text {true }}(1) /\left\{0.5 \cdot\left[1-\mathrm{FFR}^{\text {true }}(1)\right] \cdot\left[\mathrm{FFR}^{\text {true }}(2)+\mathrm{FFR}^{\text {true }}(3)\right]+\right. \\
& \text { FFR } \left.^{\text {true }}(1)\right\} \\
& \mathrm{P}_{2 \mathrm{~d}}=\mathrm{P}_{\mathrm{a}} \cdot \mathrm{FFR}^{\text {true }}(2) \cdot \mathrm{FFR}^{\text {true }}(1) /\left\{0 . 5 \cdot [ 1 - \mathrm { FFR } ^ { \text { true } } ( 1 ) ] \cdot \left[\mathrm{FFR}^{\text {true }}(2)+\right.\right. \\
& \left.\left.\operatorname{FFR}^{\text {true }}(3)\right]+\mathrm{FFR}^{\text {true }}(1)\right\} \\
& \mathrm{P}_{3 \mathrm{~d}}=\mathrm{P}_{\mathrm{a}} \cdot \mathrm{FFR}^{\text {true }}(3) \cdot \mathrm{FFR}^{\text {true }}(1) /\left\{0 . 5 \cdot [ 1 - \mathrm { FFR } ^ { \text { true } } ( 1 ) ] \cdot \left[\mathrm{FFR}^{\text {true }}(2)+\right.\right. \\
& \left.\left.\operatorname{FFR}^{\text {true }}(3)\right]+\mathrm{FFR}^{\text {true }}(1)\right\}
\end{aligned}
$$

The current condition of an artery is given by the value of its FFR $^{\text {real }}$. When $\delta$ is set to $\delta=1$, the FFR ${ }^{\text {real }}$ values for the arteries are given in terms of the measured intracoronary pressures by the following expressions:

$$
\begin{aligned}
& \operatorname{FFR}^{\text {real }}(1)=\left(P_{3 d}+P_{2 d}\right) /\left(2 \cdot P_{a}\right) \\
& F^{\text {real }}(2)=P_{2 d} / P_{a} \\
& F^{\text {real }}(3)=P_{3 d} / P_{a}
\end{aligned}
$$

If in the attempt to seek optimal resolution of the stenotic configuration the PCI practitioner intends to predict the outcome of optional revascularizations, the anticipated $\mathrm{FFR}^{\text {real }}$ values of the arteries (that reflect the condition of the arteries) need to be expressed in terms of the FFR $^{\text {true }}$ values of the arteries upon virtually exercising the optional revascularizations (see formulas (A17)-(A19) ):

$$
\mathrm{FFR}^{\text {real }}(1)=\left\{\mathrm{FFR}^{\text {true }}(1) \cdot\left[\mathrm{FFR}^{\text {true }}(3)+\mathrm{FFR}^{\text {true }}(2)\right]\right\} /\left\{\left[1-\mathrm{FFR}^{\text {true }}\right.\right.
$$
$\left.(1)] \cdot\left[F F R^{\text {true }}(2)+F F R^{\text {true }}(3)\right]+2 \cdot F^{2} R^{\text {true }}(1)\right\}$

$\mathrm{FFR}^{\text {real }}(2)=\left\{\left[2 \cdot \mathrm{FFR}^{\text {true }}(1) \cdot \mathrm{FFR}^{\text {true }}(2)\right]\right\} /\left\{\left[1-\mathrm{FFR}^{\text {true }}(1)\right] \cdot\left[\mathrm{FFR}^{\text {true }}\right.\right.$

(2) $\left.\left.+\mathrm{FFR}^{\text {true }}(3)\right]+2 \cdot \mathrm{FFR}^{\text {true }}(1)\right\}$

$$
\mathrm{FFR}^{\text {real }}(3)=\left\{\left[2 \cdot \mathrm{FFR}^{\text {true }}(1) \cdot \mathrm{FFR}^{\text {true }}(3)\right]\right\} /\left\{[ 1 - \mathrm { FFR } ^ { \text { true } } ( 1 ) ] \cdot \left[\mathrm{FFR}^{\text {true }}\right.\right.
$$

(2) $\left.\left.+\mathrm{FFR}^{\text {true }}(3)\right]+2 \cdot \mathrm{FFR}^{\text {true }}(1)\right\}$

The formulas presented in this section for the resolution of the 3-artery configuration of sizable arteries described in (Figure 1) can be also used to resolve some 2-artery configurations that happen to be equivalent to the one in (Figure 1). The prerequisite for this is that the aortic pressure is the proximal driving pressure of the 2-artery configuration. 2 common examples of this kind are the following:

\subsection{1. [stenoticproxLAD]-[stenoticremainderLAD]-[stenotic sizableD $]_{1}$}

In this scenario the first diagonal artery $D_{1}$ happens to be a sizable artery and it is potentially stenotic. The LAD artery is potentially proximally stenotic, and its proximal pressure is the aortic pressure assuming that the LMCA is non stenotic and the aortic pressure is therefore maintained all the way to the beginning of the LAD artery.

\subsection{2. [stenoticproxLCx]-[stenoticremainderLCx]-[stenoticsiz $\left.\operatorname{ableOM}_{1}\right]$}

The first obtuse marginal artery $\mathrm{OM}_{1}$ of LCx is occasionally a sizable artery that is potentially stenotic. If in this scenario LCx is potentially proximally stenotic and the LMCA is not stenotic, the aortic pressure is maintained all the way to the beginning of $\mathrm{LCx}$ artery and this is then the intracoronary proximal pressure of LCx. A numerical example of resolving a stenotic 3-artery coronary configuration by the approach and formulas of this section is presented in Section 3.1 of the article.

\subsection{Resolution of A 'Mother'-'Daughter' Scenario Using an Equivalent Stenotic 3-Artery Coronary Configuration That Includes a Small Side Branch}

As indicated already at the beginning of Section 2, if Artery 3 of the arterial configuration in (Figure 2) represents a small side branch while Artery 1 and Artery 2 are sizable arteries, $\delta$ changes completely. Its value is no longer in the proximity of $\delta=1 . \delta=\mathrm{R}_{3 \mathrm{mv}} /$ $\mathrm{R}_{2 \mathrm{mv}}$ is much greater because of the high microvascular resistance of the small side branch Artery 3 , namely $1<<\delta$. The resulting formulas presented in this section are obtained from the formulas of Section 2 by mathematically letting $\delta$ tend to infinity, $\delta \rightarrow \infty$. Calculations however show that a 3 -artery configuration with $\delta=8.58$ practically 
conforms already with the high-limit formulas [4]. It is assumed that all arteries leading to Artery 1 are stenosis-free and therefore the aortic pressure is maintained all the way to the proximal segment of Artery 1. Despite the elaborate mathematics, the final formulas employed by the PCI practitioner in the resolution of such configurations are relatively simple. In the equivalent configuration Artery 1 and Artery 2 together constitute the sizable 'mother' artery whereas Artery 3 represents the small 'daughter' side branch [7].

In the following, the formulas appropriate for the resolution of such a configuration by the PCI practitioner will be presented. As the PCI practitioner explores the outcomes of optional revascularizations, it is sometimes important to know the anticipated intracoronary pressures associated with the optional revascularizations as such an intracoronary pressure might be the driving pressure of a small side branch. The anticipated intracoronary pressures are expressed in terms of $\mathrm{FFR}^{\text {true }}$ (1), $F_{F R}^{\text {true }}(2), F^{\text {true }}(3)$ that are used as input data for the calculations involved in the explorations of the possible outcomes of those revascularizations.

$$
\begin{aligned}
& \mathrm{P}_{\mathrm{p}}=\mathrm{P}_{\mathrm{a}} \cdot \mathrm{FFR}^{\text {true }}(1) /\left\{\left[1-\mathrm{FFR}^{\text {true }}(1)\right] \cdot \mathrm{FFR}^{\text {true }}(2)+\mathrm{FFR}^{\text {true }}(1)\right\} \\
& 1<<\delta \\
& \mathrm{P}_{2 \mathrm{~d}}=\mathrm{P}_{\mathrm{a}} \cdot \mathrm{FFR}^{\text {true }}(2) \cdot \mathrm{FFR}^{\text {true }}(1) /\left\{\left[1-\mathrm{FFR}^{\text {true }}(1)\right] \cdot \mathrm{FFR}^{\text {true }}(2)+\mathrm{FFR}^{\text {true }}(1)\right\} \\
& 1<<\delta
\end{aligned}
$$

$$
\begin{aligned}
& \mathrm{P}_{3 \mathrm{~d}}=\mathrm{P}_{\mathrm{a}} \cdot \mathrm{FFR}^{\text {true }}(3) \cdot \mathrm{FFR}^{\text {true }}(1) /\left\{\left[1-\mathrm{FFR}^{\text {true }}(1)\right] \cdot \mathrm{FFR}^{\text {true }}(2)+\mathrm{FFR}^{\text {true }}(1)\right\} \\
& 1<<\delta
\end{aligned}
$$

The PCI practitioner can obtain the current values of the FFR $^{\text {true }}$ of each one of the arteries from the measured intracoronary pressures by using the following formulas [7]:

$$
\begin{aligned}
& \operatorname{FFR}^{\text {true }}(1)=1 /\left\{\left[\left(\mathrm{P}_{\mathrm{a}}-\mathrm{P}_{\mathrm{p}}\right)\right] / \mathrm{P}_{2 \mathrm{~d}}+1\right\} \\
& 1<<\delta \\
& \mathrm{FFR}^{\text {true }}(2)=\mathrm{P}_{2 \mathrm{~d}} / \mathrm{P}_{\mathrm{p}} \\
& 1<<\delta \\
& \mathrm{FFR}^{\text {true }}(3)=\mathrm{P}_{3 \mathrm{~d}} / \mathrm{P}_{\mathrm{p}} \\
& 1<<\delta
\end{aligned}
$$

The condition of an artery is determined by the value of its $F R^{\text {real }}$. Current $F_{F R}$ real of each artery can be obtained from the measured intracoronary pressures [7]:

$$
\begin{aligned}
& \operatorname{FFR}^{\text {real }}(1)=\mathrm{P}_{2 \mathrm{~d}} / \mathrm{P}_{\mathrm{a}} \\
& 1<<\delta \\
& \mathrm{FFR}^{\text {real }}(2)=\mathrm{P}_{2 \mathrm{~d}} / \mathrm{P}_{\mathrm{a}} \\
& 1<<\delta \\
& \operatorname{FFR}^{\text {real }}(3)=\mathrm{P}_{3 \mathrm{~d}} / \mathrm{P}_{\mathrm{a}}
\end{aligned}
$$

$$
1<<\delta
$$

When the PCI practitioner wants to predict the outcome of optional revascularizations of some of the 'mother'-'daughter' configurational arteries, the $\mathrm{FFR}^{\text {real }}$ of the arteries need to be expressed in terms of FFR ${ }^{\text {true }}(1), F^{2} R^{\text {true }}(2)$ and $F F R^{\text {true }}(3)$ [7]:

$$
\begin{aligned}
& \mathrm{FFR}^{\text {real }}(1)=\left\{\mathrm{FFR}^{\text {true }}(1) \cdot \mathrm{FFR}^{\text {true }}(2)\right\} / \\
& \left\{\left[1-\mathrm{FFR}^{\text {true }}(1)\right] \cdot \mathrm{FFR}^{\text {true }}(2)+\mathrm{FFR}^{\text {true }}(1)\right\} \\
& 1<<\delta \\
& \mathrm{FFR}^{\text {real }}(2)=\left\{\mathrm{FFR}^{\text {true }}(1) \cdot \mathrm{FFR}^{\text {true }}(2)\right\} / \\
& \left\{\left[1-\mathrm{FFR}^{\text {true }}(1)\right] \cdot \mathrm{FFR}^{\text {true }}(2)+\mathrm{FFR}^{\text {true }}(1)\right\} \\
& 1<<\delta \\
& \mathrm{FFR}^{\text {real }}(3)=\left\{\mathrm{FFR}^{\text {true }}(1) \cdot \mathrm{FFR}^{\text {true }}(3)\right\} / \\
& \left\{\left[1-\mathrm{FFR}^{\text {true }}(1)\right] \cdot \mathrm{FFR}^{\text {true }}(2)+\mathrm{FFR}^{\text {true }}(1)\right\} \\
& 1<<\delta
\end{aligned}
$$

It is evident that $F^{\text {real }}(1), F^{\text {real }}$ (2) and $F^{\text {real }}$ (3) have a common denominator in these expressions. This will prove useful for shortening the data processing time when optional revascularization outcomes are evaluated in PCI procedures. When using these expressions, FFR $^{\text {true }}$ of an artery that is not revascularized is identical to its original value whereas $\mathrm{FFR}^{\text {true }}$ of a revascularized artery is set to $\mathrm{FFR}^{\text {true }}=1.00$ for calculation purposes. The calculated $\mathrm{FFR}^{\text {real }}$ of the arteries reflect their condition should an optional revascularization take place.

The formulas of the multi-artery FFR method presented in this section are applicable to 'mother'-'daughter' scenarios provided that the driving pressure of the coronary configuration of (Figure 2) is the aortic pressure. It is presumed that the LMCA is not stenotic and all the arteries preceding the proximal part of the sizable 'mother' artery are not stenotic thus maintaining the aortic pressure all the way to the beginning of the 'mother' artery. Under this condition, some of the common scenarios encountered in the PCI practice for which the configuration of (Figure 2) can be regarded as an equivalent configuration, and in which the formulas of this section can be applied, are the following:

\subsection{1. [stenoticproxLAD]-[stenoticremainderLAD]-[stenotics malld $]$}

In this case the first diagonal artery $\mathrm{D}_{1}$ is a potentially stenotic small side branch. The LAD artery is potentially stenotic, and its proximal pressure is the aortic pressure. As indicated, LMCA is non stenotic thus the driving pressure of the equivalent configuration is the aortic pressure.

\subsection{2. [stenoticproxLCx]-[stenoticremainderLCx]-[ stenoticM $\left._{2}\right]$}

The second obtuse marginal artery $\mathrm{M}_{2}$ of LCx here is a potentially stenotic small side branch. In this scenario $\mathrm{LCx}$ is potentially proximally stenotic and potentially stenotic beyond the split-off 
point of $\mathrm{M}_{2}$ as well. In this case LMCA is non stenotic maintaining the aortic pressure all the way to the LCx artery.

\subsection{3. [stenoticproxRCA]-[stenoticremainderRCA]-[stenoticse condacutemarginalAM $\left.{ }_{2}\right]$}

The second acute marginal artery $\mathrm{AM}_{2}$ of RCA in this case is a potentially stenotic small side branch. Here RCA is potentially proximally stenotic and further potentially stenotic beyond the split-off point of $\mathrm{AM}_{2}$. A numerical example of the implementation of the approach and formulas of this section in the resolution of a 'mother'-'daughter' scenario is presented in Section 3.2 of the article.

\subsection{Complex 3-Artery and 4-Artery Configurations}

The stenotic coronary configuration of (Figure 3) consists of 3 sizable arteries, unprotected LMCA, LCX and LAD with an additional small first diagonal side branch $\mathrm{D}_{1}$ of LAD. Due to the substantial differences in size in this case, the sizable arteries can affect $\mathrm{D}_{1}$ through LAD but $\mathrm{D}_{1}$ cannot affect LAD nor any of the other sizable arteries. For obvious reasons, this kind of relationship constitutes a unidirectional coupling between $\mathrm{D}_{1}$ and the unprotected LMCALCx-LAD 3-artery configuration.

Chronologically, the hyperemic FFR method was first applied to a single stenotic artery with its original FFR equal to FFR ${ }^{\text {true }}$. The FFR $^{\text {true }}$ expressions for the 4 arteries under consideration, in terms of stenotic resistances and microvascular resistances associated with the arteries, are the following (Figure 3 for notation):

$$
\begin{aligned}
& \operatorname{FFR}^{\text {true }}(1)=1 /\left(1+\mathrm{R}_{1 s} / \mathrm{R}_{2 \mathrm{mv}}+\mathrm{R}_{1 \mathrm{~s}} / \mathrm{R}_{3 \mathrm{mv}}\right) ; \mathrm{FFR}^{\text {true }}(2)=1 /\left(1+\mathrm{R}_{2 s} / \mathrm{R}_{2 \mathrm{mv}}\right) \\
& \operatorname{FFR}^{\text {true }}(3)=1 /\left(1+\mathrm{R}_{3 \mathrm{~s}} / \mathrm{R}_{3 \mathrm{mv}}\right) ; \mathrm{FFR}^{\text {true }}(4)=1 /\left(1+\mathrm{R}_{4 \mathrm{~s}} / \mathrm{R}_{4 \mathrm{mv}}\right) ;
\end{aligned}
$$

\subsection{An artery within an arterial configuration}

As indicated, if a stenotic artery is just by itself, in a standalone position, the way of treatment is deduced from its value of FFR $^{\text {true }}$. However, when each of the arteries is part of a configuration of interacting stenotic arteries, the way of treatment is no longer determined by FFR ${ }^{\text {true }}$. Nevertheless, FFR ${ }^{\text {true }}$ is regarded as an important constant property characterizing the stenotic artery.

After performing intracoronary pressure measurements, the PCI practitioner should calculate the current FFR ${ }^{\text {true }}$ values for each artery in the configuration by the following formulas [6] (Figure 3):

$$
\begin{aligned}
& F_{F R}^{\text {true }}(1)=1 /\left\{\left[\left(\mathrm{P}_{\mathrm{a}}-\mathrm{P}_{\mathrm{p}}\right) \cdot 2\right] /\left(\mathrm{P}_{3 \mathrm{~d}}+\mathrm{P}_{2 \mathrm{~d}}\right)+1\right\} ; \mathrm{FFR}^{\text {true }}(2)=\mathrm{P}_{2 \mathrm{~d}} / \mathrm{P}_{\mathrm{p}} \\
& \mathrm{FFR}^{\text {true }}(3)=\mathrm{P}_{3 \mathrm{~d}} / \mathrm{P}_{\mathrm{p}} ; \mathrm{FFR}^{\text {true }}(4)=\mathrm{P}_{4 \mathrm{~d}} / \mathrm{P}_{3 \mathrm{~d}}
\end{aligned}
$$

These values characterize the arteries, however in an arterial configuration only the real FFR of an artery (denoted $\mathrm{FFR}^{\text {real) }}$, obtained by considering the actual hyperemic flow through the artery, reflects the present condition of the artery by which the way of treatment is determined.

Note that $\mathrm{FFR}^{\text {real }} \leq \mathrm{FFR}^{\text {true }}$. This inequality states the simple fact that the blood flow through a stenotic coronary artery is highest when the artery is just by itself in the absence of interconnected stenotic arteries the interference of which possibly impedes and reduces the blood flow through the artery. $\mathrm{FFR}^{\text {true }}$ therefore indicates the maximal $\mathrm{FFR}^{\text {real }}$ that the artery can have under best conditions of no interference at all and being exposed to aortic pressure. Note that this upper limit of FFR $^{\text {real }}$ may not necessarily make the condition of the artery acceptable. If the criteria of treatment of the hyperemic FFR method are followed (like in this article), FFR ${ }^{\text {true }}$ of less than 0.75 precludes the possibility of the artery to be in an acceptable condition (namely it precludes $0.75 \leq \mathrm{FFR}^{\text {real }}$ ) even under most favorable conditions, unless the artery itself is revascularized.

The next step therefore that the PCI practitioner must take in order to assess the current condition of each artery is calculating its FFR $^{\text {real }}$ from the measured intracoronary pressures by the following formulas [6,7] (see Figure 3):

$$
\begin{aligned}
& \operatorname{FFR}^{\text {real }}(1)=\left(P_{3 d}+P_{2 d}\right) /\left(P_{a} \cdot 2\right) ; \\
& \operatorname{FFR}^{\text {real }}(2)=P_{2 d} / P_{a} ; \\
& \operatorname{FFR}^{\text {real }}(3)=P_{3 d} / P_{a} ; F F R^{\text {real }}(4)=P_{4 d} / P_{a} ;
\end{aligned}
$$

If the condition of all the arteries is acceptable (namely $0.75 \leq \mathrm{FFR}^{\text {real }}$ for each artery), no further action on the part of the PCI practitioner is required unless the practitioner decides otherwise on strategic grounds, depending on the specific circumstances of the case under consideration. If $\mathrm{FFR}^{\text {real }}$ of any artery is less than 0.75 then (by the treatment criteria of the hyperemic FFR method chosen to be followed in this article) optional revascularizations should be considered. The outcomes of these optional revascularizations can be predicted from relatively simple formulas so the PCI practitioner can have an advance knowledge of the results prior to deciding about how to proceed.

In the specific scenario of Figure 3 the 3 sizable arteries rule the scene whereas the small first diagonal $\mathrm{D}_{1}$ side branch is dependent on them. Consequently, the outcome of the revascularization of sizable arteries of the configuration will be predicted first and only then the consequences for the small $\mathrm{D}_{1}$ will be calculated.

The outcomes of the optional revascularizations (in terms of FFR $^{\text {real) }}$ regarding the 3 sizable arteries, Artery 1 (LMCA), Artery 2 (LCx) and Artery 3 (LAD) can be obtained from their FFR ${ }^{\text {true }}$ values (Figure 1) [6].

In the following step the practitioner calculates the predicted outcomes of optional revascularizations for the sizable arteries:

$$
\mathrm{FFR}^{\text {real }}(1)=\left\{\mathrm{FFR}^{\text {true }}(1) \cdot\left[\mathrm{FFR}^{\text {true }}(3)+\mathrm{FFR}^{\text {true }}(2)\right]\right\} /\left\{\left[1-\mathrm{FFR}^{\text {true }}\right.\right.
$$
$\left.(1)] \cdot\left[\mathrm{FFR}^{\text {true }}(2)+\mathrm{FFR}^{\text {true }}(3)\right]+2 \cdot \mathrm{FFR}^{\text {true }}(1)\right\}$

$\mathrm{FFR}^{\text {real }}(2)=\left[2 \cdot \mathrm{FFR}^{\text {true }}(1) \cdot \mathrm{FFR}^{\text {true }}(2)\right] /\left\{\left[1-\mathrm{FFR}^{\text {true }}(1)\right] \cdot\left[\mathrm{FFR}^{\text {true }}\right.\right.$ (2) $\left.\left.+\mathrm{FFR}^{\text {true }}(3)\right]+2 \cdot \mathrm{FFR}^{\text {true }}(1)\right\}$

$\operatorname{FFR}^{\text {real }}(3)=\left[2 \cdot \mathrm{FFR}^{\text {true }}(1) \cdot \mathrm{FFR}^{\text {true }}(3)\right] /\left\{\left[1-\mathrm{FFR}^{\text {true }}(1)\right] \cdot\left[\mathrm{FFR}^{\text {true }}\right.\right.$ (2) $\left.\left.+\mathrm{FFR}^{\text {true }}(3)\right]+2 \cdot \mathrm{FFR}^{\text {true }}(1)\right\}$

In these formulas the PCI practitioner uses the current $\mathrm{FFR}^{\text {true }}$ values obtained from the measured intracoronary pressures by formulas (A45) for the arteries of the configuration. However, the 
$\mathrm{FFR}^{\text {true }}$ for any artery to be revascularized in these formulas is taken as $\mathrm{FFR}^{\text {true }}=1.00$. This is how the calculated outcomes of the optional revascularizations are explored by the practitioner in a step-by-step manner for the 3 sizable arteries until the PCI practitioner finds the route to an optimal resolution of the stenotic configuration of the sizable arteries.

It should be noted that $\mathrm{FFR}^{\text {true }}$ values of the sizable arteries, and likewise their $\mathrm{FFR}^{\text {real }}$ values, are all in principle dependent on the resistances of their associated microvasculature (see formulas (A44)). The latter are in turn dependent on stem geometry of the arteries but within the practical statistical distribution of stem geometry [5] this effect is negligible and FFR ${ }^{\text {real }}$ variation is about $\Delta F F R^{\text {real }}= \pm 0.02$ in the intermediate stenosis severity range of the arteries [4]. There is therefore no need to introduce correction terms in the formulas when going from one patient to the next.

As for the small side branch $\mathrm{D}_{1}$, since it does not influence any of the sizable arteries, this artery can be treated almost independently up to a certain point. Firstly, the PCI practitioner has to check its FFR ${ }^{\text {true }}$ (by formulas (A45)) and its current FFR ${ }^{\text {real }}$ (by formulas (A46)). Since $\mathrm{FFR}^{\text {real }} \leq \mathrm{FFR}^{\text {true }}, \mathrm{FFR}^{\text {true }}$ has to be greater than 0.75 in order for FFR real to have the chance to be greater than 0.75 if appropriate favorable conditions for $\mathrm{D}_{1}$ are created. If FFR $^{\text {true }}$ is less than 0.75 then revascularization of $D_{1}$ itself is mandatory unless its angular and diametrical limitations preclude this possibility. If, however $\mathrm{FFR}^{\text {true }}$ of $\mathrm{D}_{1}$ does not constitute an obstacle but $\mathrm{FFR}^{\text {real }}<0.75$ then the condition of $\mathrm{D}_{1}$ should be treated and the PCI practitioner has to check if stenting $\mathrm{D}_{1}$ is feasible or if any optional revascularization of the sizable arteries improves sufficiently the blood flow in $D_{1}$. In order to calculate $F F R^{\text {true }}\left(D_{1}\right)$ and $\mathrm{FFR}^{\text {real }}\left(\mathrm{D}_{1}\right)$ that would result from optional revascularizations, one needs to know first the intracoronary pressures anticipated from these revascularizations. To this end, the intracoronary pressures anticipated in each optional revascularization should be expressed in terms of the $\mathrm{FFR}^{\text {true }}$ values of the sizable arteries from formulas (A45) (Figure 1):

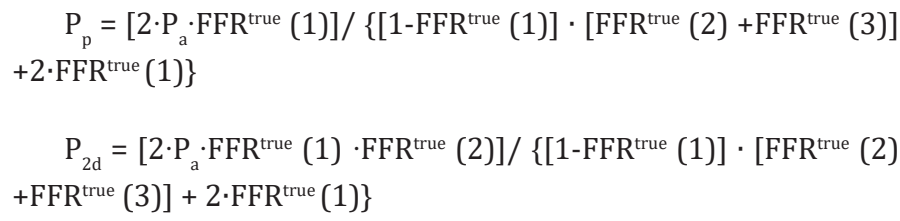

$P_{3 d}=\left[2 \cdot P_{a} \cdot F F R^{\text {true }}(1) \cdot F^{2} R^{\text {true }}(3)\right] /\left\{\left[1-F^{2} R^{\text {true }}(1)\right] \cdot\left[F F R^{\text {true }}(2)\right.\right.$ $\left.\left.+\mathrm{FFR}^{\text {true }}(3)\right]+2 \cdot \mathrm{FFR}^{\text {true }}(1)\right\}$

$$
\mathrm{P}_{4 \mathrm{~d}}=\left[2 \cdot \mathrm{P}_{\mathrm{a}} \cdot \mathrm{FFR}^{\text {true }}(1) \cdot \mathrm{FFR}^{\text {true }}(3) \cdot \mathrm{FFR}^{\text {true }}(4)\right] /\left\{\left[1-\mathrm{FFR}^{\text {true }}(1)\right] \cdot\right.
$$$$
\left.\left[\mathrm{FFR}^{\text {true }}(2)+\mathrm{FFR}^{\text {true }}(3)\right]+2 \cdot \mathrm{FFR}^{\text {true }}(1)\right\}
$$

The predicted values of $\mathrm{FFR}^{\text {true }}$ and $\mathrm{FFR}^{\text {real }}$ of $\mathrm{D}_{1}$ (Artery 4 in (Figure 3) resulting from the implementation of the optional revascularizations can be obtained in these formulas from the expressions for $\mathrm{P}_{3 \mathrm{~d}}$ and $\mathrm{P}_{4 \mathrm{~d}}$ because (by formulas (A45) and formulas (A46) respectively) one has:

$$
\operatorname{FFR}^{\text {true }}(4)=\mathrm{P}_{4 \mathrm{~d}} / \mathrm{P}_{3 \mathrm{~d}} ; \operatorname{FFR}^{\text {real }}(4)=\mathrm{P}_{4 \mathrm{~d}} / \mathrm{P}_{\mathrm{a}} \text {; }
$$

In Section 3.3 of the article an example of a resolution of a 4-artery stenotic coronary configuration (presented in (Figure 3) by the multi-artery FFR method employing the formulas of the present section and simulated intracoronary pressures is presented.

\section{References}

1. Yaeger IA (2016) A multi-artery Fractional Flow Reserve (FFR) approach for handling coronary stenosis-stenosis interaction in the multi-vessel disease (MVD) arena. Int J Cardiol 203: 807-815.

2. Fearon WF, Yong AS, Lenders G, Toth GG, Dao C, et al. (2015) The impact of downstream coronary stenosis on fractional flow reserve assessment of intermediate left main coronary artery disease: human validation. JACC Cardiovasc Interv 8: 398-403.

3. Daniels DV, van't Veer M, Pijls NHJ, van der Horst A, Yong AS, et al. (2012) The Impact of Downstream Coronary Stenoses on Fractional Flow Reserve Assessment of Intermediate Left Main Disease. JACC Cardiovasc Interv 5: 1021-1025.

4. Yaeger IA (2015) Practicability of Multi-Artery Fractional Flow Reserve (FFR) Method in the Assessment of Some Stenotic Coronary Artery Configurations in Percutaneous Coronary Intervention (PCI) Procedures. Interv Cardiol J 2(3): 31.

5. Dodge JT Jr, Brown,BG, Bolson EL, Dodge AT (1992) Lumen Diameter of Normal Human Coronary Arteries Influence of Age, Sex, Anatomic Variation,and Left Ventricular Hypertrophy or Dilation. Circulation 86: 232-246.

6. Yaeger IA (2017) Resolution of All-stenotic 3-Artery Configurations in Percutaneous Coronary Intervention (PCI) Procedures by the Multiartery Fractional Flow Reserve (FFR)Method. Cardiovasc Investig 1(1): 2.

7. Yaeger IA (2018) Resolution of coronary 'mother'-'daughter' configurations by multi-artery FFR method - a comparison with basic FFR method. Interv Cardiol 10: 65-71. 